\title{
Cauchy problem and quasi-stationary limit for the Maxwell-Landau-Lifschitz and Maxwell-Bloch equations
}

\author{
ERIC DUMAS AND FRANCK SUEUR
}

\begin{abstract}
In this paper we continue the investigation of the Maxwell-LandauLifschitz and Maxwell-Bloch equations. In particular we extend some previous results about the Cauchy problem and the quasi-stationary limit to the case where the magnetic permeability and the electric permittivity are variable.
\end{abstract}

Mathematics Subject Classification (2010): 35L45 (primary); 35Q60 (secondary).

\section{Introduction}

The models. This paper deals with two physical models which describe the propagation of electromagnetic waves, that is of the magnetic field $H$ and of the electric field $E$, in some special medium which occupies an open subset $\Omega$ of $\mathbb{R}^{3}$, with magnetic permeability $\mu$ and electric permittivity $\varepsilon$. In both cases we denote by $\bar{f}$ the extension of a function $f$ by 0 outside the set $\Omega$. The time variable is $t \geqslant 0$, and the space variable is $x \in \mathbb{R}^{3}$.

The first model refers to Maxwell-Landau-Lifschitz equations (see [10] and [28] for Physics references). The magnetic field $H$ and the electric field $E$ satisfy the Maxwell equations in $\mathbb{R}^{3}$ :

$$
\left\{\begin{array}{l}
\mu \partial_{t} H+\operatorname{curl} E=-\mu \partial_{t} \bar{M} \\
\varepsilon \partial_{t} E-\operatorname{curl} H=0 \\
\operatorname{div} \mu(H+\bar{M})=0 \\
\operatorname{div} \varepsilon E=0
\end{array}\right.
$$

where $M$ stands for the magnetic moment in the ferromagnet $\Omega$ and takes values in the unit sphere of $\mathbb{R}^{3}$. It is solution to the Landau-Lifschitz equation:

$$
\partial_{t} M=\gamma M \wedge H_{T}-\alpha M \wedge\left(M \wedge H_{T}\right) \quad \text { for } x \in \Omega,
$$

Received June 18, 2010; accepted in revised form January 20, 2011. 
where $\gamma \neq 0$ is the gyromagnetic constant, and $\alpha>0$ is some damping coefficient. Neglecting the exchange phenomenon, the total magnetic field $H_{T}$ is the sum

$$
H_{T}=H+H_{a}(\bar{M})+H_{\mathrm{ext}},
$$

where the anisotropy field writes $H_{a}(\bar{M})=\nabla_{M} \Phi(\bar{M})$, for some convex function $\Phi$, and $H_{\text {ext }}$ is some applied (exterior) magnetic field.

The second model refers to Maxwell-Bloch equations (see for example [7,8, $16,35,38,41])$. In this setting $\Omega$ denotes some quantum medium with $N \in \mathbb{N}$ energy levels described by a Hermitian, non-negative, $N \times N$ density matrix $\rho$. Assuming the usual dipolar approximation, these quantum states change under the action of an electric field $E$ by the quantum Liouville-Von Neumann (or Bloch) equation:

$$
i \partial_{t} \rho=[\Lambda-E \cdot \Gamma, \rho]+i Q(\rho) .
$$

The $N \times N$ Hermitian symmetric matrix $\Lambda$, with entries in $\mathbb{C}$, represents the (electromagnetic field-) free Hamiltonian of the medium. The dipole moment operator $\Gamma$ is a $N \times N$ Hermitian matrix, with entries in $\mathbb{C}^{3}$, and depends on the material considered. The (linear) relaxation term $Q(\rho)$ takes dissipative effects into account (see $[5,6,32])$. The polarization $P$ of the matter is given by the constitutive law $P=\operatorname{Tr}(\Gamma \rho)$ which influences back the electric field $E$. Again, the electromagnetic field satisfies the Maxwell equations in $\mathbb{R}^{3}$ :

$$
\left\{\begin{array}{l}
\mu \partial_{t} H+\operatorname{curl} E=0, \\
\varepsilon \partial_{t} E-\operatorname{curl} H=-\partial_{t} \bar{P}, \\
\operatorname{div}(\varepsilon E+\bar{P})=0, \\
\operatorname{div} \mu H=0 .
\end{array}\right.
$$

Cauchy problems. We first address the questions of global existence, uniqueness and stability for the Cauchy problem associated with these equations. The physically relevant solutions have finite energy: they satisfy the usual $\left(L^{2}\right)$ energy estimates. Mathematically, this regularity leads to weak solutions and is usually not enough to ensure the desired uniqueness and stability properties (requiring for these hyperbolic semilinear systems in space dimension 3, in the general theory, $H^{s}$ Sobolev regularity with $s>3 / 2$ ).

However, in the case of the Maxwell-Landau-Lifschitz system, Joly, Métivier and Rauch [24] noticed that specific (algebraic) properties of the nonlinearities, as well as (geometric) properties of the differential operator involved, allowed to show the existence of global finite energy solutions (essentially, using compensated compactness arguments) enjoying stability properties. Furthermore, only a small amount of regularity (curl $H$ and $\operatorname{curl} E$ in $L^{2}$ ) ensures uniqueness. This is achieved using dispersive properties of the system; namely, a limit Strichartz estimate controlling the $L_{t}^{2} L_{x}^{\infty}$ norm of (a limited frequency part of) the fields $H$ and $E$. These results were obtained for equations posed in the whole space $\left(\Omega=\mathbb{R}^{3}\right)$ and for constant coefficients $\varepsilon$ and $\mu$. 
In practice, the various coefficients of the system may not be constant. Typically, the magnetic permeability and electric permittivity may depend on the space variable $x$ and have jumps across the boundary of the domain $\Omega$.

Adapting the above mentioned compensated compactness argument, Jochmann established in [23] the existence and weak stability of global finite energy solutions for the Maxwell-Landau-Lifschitz system, considering any domain $\Omega \subset \mathbb{R}^{3}$, and variable, possibly discontinuous coefficients $\left(\varepsilon, \mu \in L^{\infty}\left(\mathbb{R}^{3}\right)\right)$. In the (space) 2dimensional case, we refer to the work of Haddar [21].

Concerning the Maxwell-Bloch system, the first author noticed that it shares with the Maxwell-Landau-Lifschitz some of its structural properties. This author thus showed in [18] results on existence and uniqueness of global finite energy solutions, similar to the ones of Joly, Métivier and Rauch, but for some general class of systems including the two models above. Again, these results where obtained for equations posed in the whole space and for constant coefficients $\varepsilon$ and $\mu$.

Here, we continue this study, again for a general class of systems including the Maxwell-Landau-Lifschitz equations and the Maxwell-Bloch equations, so as to enlight the similarities and differences between these two models. Adapting Jochmann's method, we show the existence and stability of global finite energy solutions, for a given domain $\Omega \subset \mathbb{R}^{3}$, and $L^{\infty}$ coefficients. Then, for smooth coefficients, constant out of some compact set, we prove a limit Strichartz estimate analogous to the one obtained by Joly, Métivier and Rauch in the constant coefficient case. This allows us to show propagation of regularity and uniqueness when initially, curl $H$ and curl $E$ belong to $L^{2}\left(\mathbb{R}^{3}\right)$. As a corollary of a result of Saint-Raymond [40], we also infer generic uniqueness of the global finite energy solutions.

Quasi-stationary limits. Next, we turn to the problem of the so-called quasistationary limit. Physically, this regime appears when the domain $\Omega$ is small compared to the wavelength. Mathematically, it amounts to some long-time asymptotics (replacing in the equations $\partial_{t}$ by $\eta \partial_{t}$, for some small parameter $\eta$ ) with weak nonlinearities (also scaled so as to have an amplitude of size $\eta$ ).

Jochmann showed in [23] the weak convergence of the corresponding solutions to the Maxwell-Landau-Lifschitz system towards the solutions of some reduced system driven by the magnetization, using the weak stability property. Starynkevitch extended this result, proving strong and global-in-time convergence in the constant coefficient case in [42], thanks to local energy estimates performed on the explicit fundamental solution of the associated wave equation. He also obtained the same result in the case of smooth coefficients, constant out of some compact set, in [43], thanks to dispersive estimates obtained from resolvent estimates on elliptic operators.

Here, we apply the same methods to our general systems to get weak and strong convergence in the quasi-stationary limit. For the latter however, some time integrability assumption is needed to conclude, which is satisfied by the MaxwellLandau-Lifschitz system (since $\partial_{t} M \in L^{2}((0, \infty) \times \Omega)$ ), but we do not know if the Maxwell-Bloch system enjoys such a property. 
Remark 1.1. Taking exchange energy into account, one should add to the total magnetic field in (1.3) a term $-K \Delta M$ with $K>0$. The resulting system is then parabolic. We refer to [1,2,12-14] and [45] for works on the corresponding (weak or strong) Cauchy problem, long-time asymptotics and quasi-stationary limits.

\section{Main results}

Let us stress that we do not assume that $\Omega$ is bounded, for the moment. To deal with both the Maxwell-Landau-Lifschitz system (1.1)-(1.2) and the Maxwell-Bloch system (1.4)-(1.5), we put these two models above into a single class of systems consisting in the coupling of the Maxwell equations (with the fields $H$ and $E$ as unknowns) with some ODE (corresponding to a third unknown variable). The resulting sytem is symmetrizable hyperbolic, with semilinear nonlinearity, and some structure assumptions are made, such as affine dependence of the nonlinearity with respect to the electromagnetic field, and a priori pointwise estimates on the third unknown variable. One of the key points in our study is that the electromagnetic fields decompose into an "irrotational" part, which is directly related to this third unknown, and a "divergence free" part, which solves some wave equation.

\subsection{An abstract setting}

On any finite-dimensional vector space $\mathbb{R}^{N}$, we denote by $u \cdot u^{\prime}$ the usual scalar product between vectors $u$ and $u^{\prime}$, and by $|\cdot|$ the associated norm. For all $r>0$, $B_{r}$ denotes the (closed) ball centered at 0 , with radius $r$.

We consider two scalar functions $\kappa_{1}(x)$ and $\kappa_{2}(x)$, which are uniformly positive:

$$
\text { for } i=1,2, \quad \kappa_{i} \in L^{\infty}\left(\mathbb{R}^{3}\right), \quad \text { and } \quad \exists c>0, \kappa_{i} \geqslant c .
$$

We denote by $H_{\text {curl }}$ the space of functions $f$ in $L^{2}\left(\mathbb{R}^{3}, \mathbb{R}^{3}\right)$ with curl $f$ in $L^{2}\left(\mathbb{R}^{3}, \mathbb{R}^{3}\right)$. We consider the operator $B$ defined by

$$
B\left(u_{1}, u_{2}\right)=\left(\kappa_{1}^{-1} \operatorname{curl} u_{2},-\kappa_{2}^{-1} \operatorname{curl} u_{1}\right) \text { for } u:=\left(u_{1}, u_{2}\right) \in D(B):=H_{\text {curl }} \times H_{\text {curl }} .
$$

This is a skew self-adjoint operator on the Hilbert space $L^{2}\left(\mathbb{R}^{3}, \mathbb{R}^{6}\right)$ endowed with the scalar product

$$
\left\langle\left(u_{1}, u_{2}\right),\left(u_{1}^{\prime}, u_{2}^{\prime}\right)\right\rangle_{\kappa_{1}, \kappa_{2}}:=\int_{\mathbb{R}^{3}}\left(\kappa_{1} u_{1} \cdot u_{1}^{\prime}+\kappa_{2} u_{2} \cdot u_{2}^{\prime}\right) d x .
$$

We denote by $P\left(u_{1}, u_{2}\right):=\left(P_{1} u_{1}, P_{2} u_{2}\right)$ the orthogonal projector on $(\operatorname{ker} B)^{\perp}$ with respect to the weighted scalar product above, so that for $i=1,2$,

$$
\begin{aligned}
& \operatorname{ran} P_{i}=\left\{u_{i} \in L^{2}\left(\mathbb{R}^{3}, \mathbb{R}^{3}\right) \mid \operatorname{div}\left(\kappa_{i} u_{i}\right)=0\right\}, \\
& \operatorname{ran}\left(I d-P_{i}\right)=\left\{u_{i} \in L^{2}\left(\mathbb{R}^{3}, \mathbb{R}^{3}\right) \mid \operatorname{curl}\left(u_{i}\right)=0\right\} .
\end{aligned}
$$


We consider a function $F: \mathbb{R}^{3} \times \mathbb{R}^{d} \times \mathbb{R}^{6} \rightarrow \mathbb{R}^{d}$, where $d \in \mathbb{N}$, affine in its third variable, and written

$$
F(x, v, u)=F_{0}(x, v)+F_{1}(x, v) u .
$$

For each $j=0,1, F_{j}$ is measurable with respect to $x$ and continuously differentiable with respect to $v$. Furthermore,

$$
\begin{aligned}
\text { for } j=0,1, & \text { for almost all } x \in \mathbb{R}^{3}, F_{j}(x, 0)=0, \\
\text { and } \quad & \forall R>0, \text { for almost all } x \in \mathbb{R}^{3}, \forall v \in B_{R}, \\
& \left|F_{j}(x, v)\right|+\left|\partial_{v} F_{j}(x, v)\right| \leqslant C_{F}(R) .
\end{aligned}
$$

Finally, we assume that there exists $K \geqslant 0$ such that:

$$
\text { for almost all } x \in \mathbb{R}^{3}, \forall(v, u) \in \mathbb{R}^{d} \times \mathbb{R}^{6}, \quad F(x, v, u) \cdot v \leqslant K|v|^{2} .
$$

Remark 2.1. The constant $K$ above may sometimes be taken equal to zero. In this case, Estimate (i) in Theorem 2.6 is improved, since $v$ does not undergo any growth. This is the case for the Maxwell-Landau-Lifschitz model, as well as for the Maxwell-Bloch model, when only transverse relaxation is taken into account $\left(Q(\rho)=-\gamma \rho_{o d}\right.$, for some $\gamma \geqslant 0$, and with $\rho_{o d}$ the off-diagonal part of $\left.\rho\right)$.

We also consider a function $l=\left(l_{1}, l_{2}\right) \in\left(L^{\infty}\left(\mathbb{R}^{3}, L\left(\mathbb{R}^{d}, \mathbb{R}^{3}\right)\right)\right)^{2}$, where $L\left(\mathbb{R}^{d}, \mathbb{R}^{3}\right)$ denotes the space of linear functions from $\mathbb{R}^{d}$ to $\mathbb{R}^{3}$. We introduce the following shorthand notation: for any $x \in \mathbb{R}^{3},\left(\kappa^{-1} \cdot l\right)(x)$ is the mapping from $\mathbb{R}^{d}$ to $\mathbb{R}^{6}$, such that

for almost all $x \in \mathbb{R}^{3}, \forall v \in \mathbb{R}^{d}, \quad\left(\kappa^{-1} \cdot l\right)(x) v:=\left(\kappa_{1}(x)^{-1} l_{1}(x) v, \kappa_{2}(x)^{-1} l_{2}(x) v\right)$.

Then, for any $U:=(u, v)$ in

$$
\mathbf{L}^{2}:=L^{2}\left(\mathbb{R}^{3}, \mathbb{R}^{6}\right) \times L^{2}\left(\Omega, \mathbb{R}^{d}\right),
$$

the conditions

$$
\operatorname{div}\left(\kappa_{1} u_{1}-l_{1} \bar{v}\right)=0, \quad \operatorname{div}\left(\kappa_{2} u_{2}-l_{2} \bar{v}\right)=0,
$$

may be equivalently written

$$
(I d-P)\left(u-\left(\kappa^{-1} \cdot l\right) \bar{v}\right)=0 .
$$

We look for $U \in C\left([0, \infty), \mathbf{L}^{2}\right)$, solution to

$$
\begin{aligned}
\left(\partial_{t}+B\right) u & =\left(\kappa^{-1} \cdot l\right) F(x, \bar{v}, u) & & \text { for } x \in \mathbb{R}^{3}, \\
\partial_{t} v & =F(x, v, u) & & \text { for } x \in \Omega,
\end{aligned}
$$

and (2.6). We recall that for any function $f$ defined on $\Omega$, we denote by $\bar{f}$ the extension of $f$ (to $\mathbb{R}^{3}$ ) by 0 outside $\Omega$. We also impose the condition

$$
v \in L_{\mathrm{loc}}^{\infty}\left((0, \infty), L^{\infty}\left(\Omega, \mathbb{R}^{d}\right)\right) .
$$

Equations (2.6)-(2.8) are understood in the distributional sense, noticing that (2.9) gives sense to the nonlinear term, since the function $F(x, v, u)$ is affine in $u$. 
Remark 2.2. Equations (2.6)-(2.8) reduce to the Maxwell-Landau-Lifschitz system (1.1)-(1.2) when $u_{1}=H, u_{2}=E, v=M$ (with $d=3$ ), $\kappa_{1}=\mu, \kappa_{2}=\varepsilon, l_{1}=$ $-\mu, l_{2}=0, F(x, v, u)=\gamma v \wedge\left(u_{1}+H_{a}(v)+H_{\mathrm{ext}}\right)-\alpha v \wedge\left(v \wedge\left(u_{1}+H_{a}(v)+H_{\mathrm{ext}}\right)\right)$ and to the Maxwell Bloch system (1.4)-(1.5) when $u_{1}=H, u_{2}=E, v=\rho$ (with $\left.d=N^{2}\right), \kappa_{1}=\mu, \kappa_{2}=\varepsilon, l_{1}=0, l_{2}=\operatorname{Tr}(\Gamma \cdot), F(x, v, u)=-i\left[\Lambda-u_{2}\right.$. $\Gamma, v]+Q(v)$. The exterior magnetic field above is usually depending on time. We did not consider such time-dependent coefficients in our study, since it would have made notations more intricate; up to some integrability assumptions, this extension is straightforward.

Definition 2.3. We call $U=(u, v) \in C\left([0, \infty), \mathbf{L}^{2}\right)$ a global finite energy solution to (2.6)-(2.8) if (2.9) holds true and $U$ is a solution to (2.6)-(2.8) in the distributional sense.

Remark 2.4. Equation (2.6) has to be seen as a (linear) constraint, which propagates from $t=0$ for solutions to (2.7)-(2.8):

$$
\partial_{t}(I d-P)\left(u-\left(\kappa^{-1} \cdot l\right) \bar{v}\right)=0 .
$$

Indeed, by definition of the projector $P$, we have $(I d-P) B=0$, so that we get (2.10) when applying $(I d-P)$ to (2.7), using (2.8) (which extends to all $x \in \mathbb{R}^{3}$ since $F(x, 0, u) \equiv 0)$ and commuting the derivative $\partial_{t}$ with $(I d-P)$ and $\kappa^{-1} \cdot l$.

We therefore have to consider initial data $U_{\text {init }}$ satisfying (2.6), and for such constrained initial data, the solutions to (2.7)-(2.8) also satisfy (2.6) as long as they exist. We shall write $U_{\text {init }}:=\left(u_{\text {init }}, v_{\text {init }}\right)$ with $u_{\text {init }}:=\left(u_{\text {init, } 1}, u_{\text {init, } 2}\right)$.

Definition 2.5. Let $L_{\text {div }}$ be the set of functions $U:=(u, v) \in L^{2}\left(\mathbb{R}^{3}, \mathbb{R}^{6}\right) \times$ $\left(L^{2}\left(\Omega, \mathbb{R}^{d}\right) \cap L^{\infty}\left(\Omega, \mathbb{R}^{d}\right)\right)$ satisfying (2.6).

\subsection{Cauchy problems}

Our first result states the existence of global finite energy solutions to (2.6)-(2.8).

Theorem 2.6. Assume (2.1) and (2.3)-(2.5). For any $U_{\text {init }}$ in $L_{\mathrm{div}}$, there exists $U:=$ $(u, v) \in C\left([0, \infty), \mathbf{L}^{2}\right)$, global finite energy solution to $(2.6)-(2.8)$ with $U_{\text {init }}$ as initial data. Moreover, for all $T>0$, there is $C=C\left(T, F, l,\left\|v_{\text {init }}\right\|_{L^{\infty}}\right)$ such that

(i) for almost all $x \in \mathbb{R}^{3}$, for all $t \geqslant 0,|v(t, x)| \leqslant\left|v_{\text {init }}(x)\right| e^{K t}$ (with $K$ from (2.5));

(ii) for all $t \in[0, T],\|(u, v)(t)\|_{\mathbf{L}^{2}} \leqslant C\left\|U_{\text {init }}\right\|_{\mathbf{L}^{2}}$;

(iii) $v \in W_{\mathrm{loc}}^{1, \infty}\left((0, \infty), L^{2}\left(\Omega, \mathbb{R}^{d}\right)\right)$, and for almost every $t \in[0, T],\left\|\partial_{t} v(t)\right\|_{L^{2}(\Omega)} \leqslant$ $C\left\|U_{\text {init }}\right\|_{\mathbf{L}^{2}}$.

Finally, if $\mathcal{U}_{\text {init }}$ is a bounded set of $L_{\mathrm{div}}$ which is compact in $\mathbf{L}^{2}$, then for all $T>0$, the set $\mathcal{U}$ of the above solutions with Cauchy data in $\mathcal{U}_{\text {init }}$ is compact in $C\left([0, T], \mathbf{L}^{2}\right)$. 
To establish this first result, we follow the strategy of Jochmann in [23], which is itself an improvement of the method by Joly, Métivier and Rauch in [24]. This is the classical regularization method, in which (global-in-time) approximate solutions $U^{n}=\left(u^{n}, v^{n}\right)$ are built first (Section 3.2); the delicate step consists of course in passing to the limit $n \rightarrow \infty$ in the regularization (Section 3.3). Pointwise bounds are available for $v^{n}$, which imply $L^{p}$ bounds for $(I d-P) u^{n}=(I d-P)\left(\kappa^{-1} \cdot l\right) \overline{v^{n}}$, for finite $p$. The main argument relies on compensated compactness, applied to $P u^{n}$ (Lemma 3.4).

As a byproduct of the proof of Theorem 2.6, we also have the following version of stability, where we assume strong convergence only for the $v$ part of the initial data. It will be useful below ( $c f$. proof of Theorem 2.10) when considering the weak quasi-stationary limit.

Proposition 2.7. Let $\left(U^{n}\right)_{n \in \mathbb{N}}$ be a sequence in $L_{\mathrm{loc}}^{\infty}\left((0, \infty), L_{\mathrm{div}}\right)$, bounded in $L_{\mathrm{loc}}^{\infty}\left((0, \infty), \mathbf{L}^{2}\right)$, with $\left(v^{n}\right)_{n \in \mathbb{N}}$ bounded in $W_{\mathrm{loc}}^{1, \infty}\left((0, \infty), L^{2}(\Omega)\right) \cap L_{\mathrm{loc}}^{\infty}((0, \infty)$, $\left.L^{\infty}(\Omega)\right)$ satisfying $(2.8),\left.v^{n}\right|_{t=0} \rightarrow v_{\text {init }}$ in $L^{2}(\Omega)$ and $B u^{n}=\partial_{t} D^{n}$ with $\left(D^{n}\right)_{n}$ bounded in $L_{\mathrm{loc}}^{\infty}\left((0, \infty), L^{2}\left(\mathbb{R}^{3}\right)\right)$. Then, up to a subsequence, $v^{n}$ converges to $v$ in $L_{\mathrm{loc}}^{\infty}\left((0, \infty), L^{p}(\Omega)\right)$ for any $p \geqslant 2$ and in $L_{\mathrm{loc}}^{\infty}\left((0, \infty), L^{\infty}(\Omega)\right)$ weak $*, u^{n}$ converges to $u$ in $L_{\mathrm{loc}}^{\infty}\left((0, \infty), L^{2}\left(\mathbb{R}^{3}\right)\right)$ weak $*$, and $U:=(u, v)$ satisfies $(2.6),(2.8)$, as well as $\left.v\right|_{t=0}=v_{\text {init. }}$

Let us now turn our atttention to smoother solutions. We need to assume more smoothness on the coefficients $\varepsilon$ and $\mu$. Of course, when considering in some physical situation a domain $\Omega$ with boundaries, the coefficients $\varepsilon$ and $\mu$ experiment discontinuity jumps. Since we do not know how to tackle this physical case, we assume from now on that

$\Omega$ is bounded, $\quad$ and with $\mathcal{K}=\bar{\Omega}, \quad \kappa_{i}-1 \in \mathcal{C}_{\mathcal{K}}^{\infty}\left(\mathbb{R}^{3}\right), i=1,2$,

where $\mathcal{C}_{\mathcal{K}}^{\infty}\left(\mathbb{R}^{3}\right)$ is the space of $\mathcal{C}^{\infty}$ functions on $\mathbb{R}^{3}$, with support contained in $\mathcal{K}$. In order to get a uniqueness result, we only need to ensure that the "divergence free" part $P u$ of the fields has the $H^{1}$ regularity. To this end, once a finite energy solution is given, we make use of the linear system solved by $P u$, with coefficients depending on the rest of the solution. As in [24], we proceed in two steps: we begin with the propagation of $H^{\mu}$ regularity, for $\mu \in(0,1)$, using Strichartz estimates (Proposition 4.6). Applying this result with $\mu=1 / 2$ provides enough integrability for the coefficients of the above mentioned linear equation to ensure propagation of $H^{1}$ regularity. This implies that $u$ is "almost" $L^{\infty}$, a natural condition to prove uniqueness of the solution. Technically, a $L^{\infty}$ approximation of $u$ is built thanks to a limit Strichartz estimate for low frequencies (Proposition 4.7). We also need a decoupling assumption, which was introduced in [18], and is satisfied by the Maxwell-Landau-Lifschitz system as well as by the Maxwell-Bloch system. 
Theorem 2.8. In addition to the assumptions of Theorem 2.6, assume (2.11). Let $\mu \in] 0,1]$, and $U_{\text {init }} \in L_{\text {div }}$ with curl $u_{\text {init }, i} \in H^{\mu-1}\left(\mathbb{R}^{3}\right)$, for $i=1$, 2. Then, the following holds true:

(a) Any solution $U$ to (2.6)-(2.8) with $U_{\text {init }}$ as initial data given by Theorem 2.6 satisfies curl $u_{i} \in C\left([0, \infty], H^{\mu-1}\left(\mathbb{R}^{3}\right)\right)$, for $i=1,2$.

(b) If $\mu=1$, assuming moreover that

$$
\begin{aligned}
& \text { there exists } j \in\{1,2\} \text { such that } l_{3-j} F=0 \\
& \text { and such that } F \text { depends only on }\left(x, v, u_{j}\right) \text {, }
\end{aligned}
$$

there exists only one solution to (2.6)-(2.8) with $U_{\text {init }}$ as initial data as in Theorem 2.6.

Theorem 2.8 asserts that the uniqueness property holds for initial data $U_{\text {init }}$ in $L_{\text {div }}$ with curl $u_{\text {init, } i} \in L^{2}\left(\mathbb{R}^{3}\right), i=1,2$, which are dense in $L_{\text {div }}$ for the topology of $\mathbf{L}^{2}$. The following theorem says that the uniqueness property even holds generically for the following topologies. Let $\tau_{s}$ and $\tau_{w}$ denote respectively the strong and weak topologies of $L^{2}\left(\mathbb{R}^{3}, \mathbb{R}^{6}\right)$ and let $\tilde{\tau}_{s}$ denote the strong topology of $L^{2}\left(\Omega, \mathbb{R}^{d}\right)$. We consider the product topology $\tau_{s s}$ (respectively $\tau_{w s}$ ) on $\mathbf{L}^{2}$ obtained from $\tau_{s}$ (respectively $\tau_{w}$ ) and $\tilde{\tau}_{s}$.

Theorem 2.9. Under the assumptions of Theorem 2.8 (b), for any $C_{\text {init }}>0$, there exists $a G_{\delta}$ dense set $\widetilde{L_{\mathrm{div}}}$ in the set $\left\{U_{\mathrm{init}} \in L_{\mathrm{div}} \mid\left\|v_{\mathrm{init}}\right\|_{L^{\infty}(\Omega)} \leqslant C_{\mathrm{init}}\right\}$ for the topology $\tau_{s s}$ and $\tau_{w s}$, such that for any $U_{\text {init }} \in \widetilde{L_{\mathrm{div}}}$, there exists only one solution to (2.6)-(2.8) with $U_{\text {init }}$ as initial data, with the same properties as in Theorem 2.6.

Let us stress that we cannot expect that the problem (2.6)-(2.8) admits smoother solutions than the ones given by Theorem 2.8 since, by definition, $\bar{v}$ is discontinuous across the boundary $\partial \Omega$. However it follows from the general theory of discontinuous solutions of hyperbolic semilinear systems [34,39] that the problem (2.6)-(2.8) admits piecewise regular solutions discontinuous accross $\partial \Omega$ (let us also refer to the appendix of [44]). Yet the general theory only guaranties local-in-time solutions. We do not know if in the particular case of the problem (2.6)-(2.8) global-in-time solutions can be obtained.

\subsection{Quasi-stationary limits}

As described in the Introduction, the quasi-stationary regime consists in the limit $\eta \rightarrow 0^{+}$for (2.6)-(2.8), where $\partial_{t}$ is replaced with $\eta \partial_{t}$, and $F$ is replaced with $\eta F$. Equations (2.6) and (2.8) are invariant under this rescaling, whereas (2.7) becomes

$$
\left(\eta \partial_{t}+B\right) u=\eta\left(\kappa^{-1} \cdot l\right) F(x, \bar{v}, u), \quad \text { for } x \in \mathbb{R}^{3} .
$$

For this semi-classical version of (2.8), it is still true that the constraint (2.6) is propagated from the initial data. Formally, in the limit $\eta \rightarrow 0^{+}, v$ still satisfies 
(2.8), whereas $u$ satifies (2.6) and $B u=0$. But for $U=(u, v) \in C\left([0, \infty), \mathbf{L}^{2}\right)$, these last two conditions are equivalent to the fact that for all $t \geqslant 0, u(t)$ is directly determined by $v(t)$, and more precisely:

$$
u=(I d-P) u=(I d-P)\left(\kappa^{-1} \cdot l\right) \bar{v},
$$

with $P$ from (2.2). Then, (2.8) becomes

$$
\partial_{t} v=F\left(x, v,\left((I d-P)\left(\kappa^{-1} \cdot l\right) \bar{v}\right)_{\left.\right|_{\Omega}}\right) .
$$

Using the stability result given by Proposition 2.7, we have a first result of convergence towards the quasi-stationary limit, weakly for $u$ and locally in time for $v$ :

Theorem 2.10. Assume (2.1) and (2.3)-(2.5). For any $U_{\text {init }}$ in $L_{\mathrm{div}}$, for any $\eta \in(0,1)$, let $U^{\eta}:=\left(u^{\eta}, v^{\eta}\right)$ be a global finite energy solution to (2.6), (2.8) and (2.13) with $U_{\text {init }}$ as initial data. Then, up to a subsequence, $v^{\eta}$ converges in $L_{\mathrm{loc}}^{\infty}\left((0, \infty), L^{p}(\Omega)\right)$ for all $p \geqslant 2$ and in $L_{\mathrm{loc}}^{\infty}\left((0, \infty), L^{\infty}(\Omega)\right)$ weak $*$ towards a solution $v$ to (2.15), with $v_{\text {init }}$ as initial data; $P u^{\eta}$ converges to 0 in $L_{\mathrm{loc}}^{\infty}\left((0, \infty), L^{2}(\Omega)\right)$ weak $*$, and $(I d-P) u^{\eta}=(I d-P)\left(\kappa^{-1} \cdot l\right) \overline{v^{\eta}}$ converges in $L_{\text {loc }}^{\infty}\left((0, \infty), L^{p}\left(\mathbb{R}^{3}\right)\right)$ for all $p \geqslant 2$ towards $u$, given by $(2.14)$.

Convergence of the whole sequence $U^{\eta}$ is ensured as soon as the Cauchy problem associated with the limiting equation (2.15) has a unique solution. This is given by the following proposition, which extends [43, Theorem 3.1] by Starynkevitch.

Proposition 2.11. Assume (2.11), and let $v_{\text {init }} \in L^{\infty}(\Omega)$. Then, there is a unique $v \in C\left([0, \infty), L^{2}(\Omega)\right) \cap L_{\mathrm{loc}}^{\infty}\left((0, \infty), L^{\infty}(\Omega)\right)$ solution to $(2.15)$, with $v_{\text {init }}$ as initial data.

We also prove strong and global-in-time convergence for $u$, assuming (2.11) again, as well as integrability in time for $\left\|\partial_{t} v\right\|_{L^{2}(\Omega)}^{2}$ and non-trapping for some wave operator:

Theorem 2.12. Under the assumptions of Theorem 2.10, assume moreover (2.11), the non-trapping hypothesis (6.6) and that $\partial_{t} v^{\eta}$ is bounded (with respect to $\eta$ ) in $L^{2}((0, \infty) \times \Omega)$. Then, $P u^{\eta}$ goes to zero in $L^{2}\left((0, \infty), L_{\text {loc }}^{2}\left(\mathbb{R}^{3}\right)\right)$.

Remark 2.13. In the case of the Maxwell-Landau-Lifschitz system(1.1)-(1.2), $\partial_{t} M$ actually belongs to $L^{2}((0, \infty) \times \Omega)$. Define the energy $\mathcal{E}(t)$ as

$$
\mathcal{E}(t)=\frac{1}{2} \int_{\mathbb{R}^{3}}\left(\varepsilon|E|^{2}+\mu|H|^{2}\right) d x+\int_{\Omega} \mu\left(\Phi(M)+\frac{1}{2}\left|H_{\mathrm{ext}}-M\right|^{2}\right) d x .
$$

Differentiating formally this expression with respect to time, we see that the integral of $H \cdot \operatorname{curl} E-E \cdot \operatorname{curl} H=\operatorname{div}(E \wedge H)$ vanishes, as well as $M \cdot \partial_{t} M$ (since $|M|$ is constant). Using the orthogonality relations of the nonlinearity, we get

$$
\partial_{t} M \cdot H_{T}=\alpha\left|M \wedge H_{T}\right|^{2} \text { and }\left|\partial_{t} M\right|^{2}=\left(\alpha^{2}+\gamma^{2}\right)\left|M \wedge H_{T}\right|^{2},
$$


so that estimate (ii) in Theorem 2.6 is improved to

$$
\begin{aligned}
\mathcal{E}(t) & +\frac{\alpha}{\alpha^{2}+\gamma^{2}} \int_{0}^{t}\left\|\sqrt{\mu} \partial_{t} M\left(t^{\prime}\right)\right\|_{L^{2}(\Omega)}^{2} d t^{\prime}-\frac{1}{2}\left\|\sqrt{\mu} H_{\mathrm{ext}}(t)\right\|_{L^{2}(\Omega)}^{2} \\
& +\int_{0}^{t}\left(\int_{\Omega} \mu M \cdot \partial_{t} H_{\mathrm{ext}} d x\right) d t^{\prime}=c s t,
\end{aligned}
$$

and the same is true with the quasi-stationary scaling. Assuming for example that $H_{\text {ext }} \in L_{t}^{\infty} L_{x}^{2}$ and $\partial_{t} H_{\text {ext }} \in L_{t, x}^{1}$, we deduce that $\mathcal{E}$ is bounded, and $\partial_{t} M$ belongs to $L^{2}((0, \infty) \times \Omega)$.

In the case of the Maxwell-Bloch system, we do not know if such an estimate is available for $\partial_{t} \rho$.

\section{Existence of global finite energy solutions: proof of Theorem 2.6}

\subsection{Technical interlude 1}

\subsubsection{Intersections and sums of Banach spaces}

We recall some useful properties of the intersection and the sum of Banach spaces. Consider two Banach spaces $X_{1}$ and $X_{2}$ that are subsets of a Hausdorff topological vector space $X$. Then

$$
\begin{aligned}
& X_{1} \cap X_{2}:=\left\{f \in X \mid f \in X_{1}, f \in X_{2}\right\} \\
& \text { (respectively } X_{1}+X_{2}:=\{f \in X \mid E(f) \neq \emptyset\}, \\
& \text { where } E(f):=\left\{\left(f_{1}, f_{2}\right) \in X_{1} \times X_{2} \mid f_{1}+f_{2}=f\right\} \text { ) }
\end{aligned}
$$

is a Banach space endowed with the norm

$$
\begin{aligned}
&\|f\|_{X_{1} \cap X_{2}}:=\|f\|_{X_{1}}+\|f\|_{X_{2}} \\
&\text { (respectively } \left.\|f\|_{X_{1}+X_{2}}:=\inf \left\{\left\|f_{1}\right\|_{X_{1}}+\left\|f_{2}\right\|_{X_{2}} \mid\left(f_{1}, f_{2}\right) \in E(f)\right\}\right) .
\end{aligned}
$$

If furthermore $X_{1} \cap X_{2}$ is a dense subset of both $X_{1}$ and $X_{2}$, then $\left(X_{1} \cap X_{2}\right)^{\prime}=$ $X_{1}^{\prime}+X_{2}^{\prime}$ and $\left(X_{1}+X_{2}\right)^{\prime}=X_{1}^{\prime} \cap X_{2}^{\prime}(c f$. Bergh and Löfström [4, Lemma 2.3.1 and Theorem 2.7.1]).

\subsubsection{Mollifiers}

We shall use the following symmetric operators $R^{n}: L^{2}\left(\mathbb{R}^{3}\right) \rightarrow L^{2}\left(\mathbb{R}^{3}\right)$, defined by

$$
\left(R^{n} f\right)(x):=\int_{\mathbb{R}^{3}} f(y) w^{n}(x-y) d y \quad \text { for } x \in \mathbb{R}^{3},
$$


where $w^{n} \in C_{0}^{\infty}\left(\mathbb{R}^{3}\right)$ is a mollifier with supp $w^{n} \subset B(0,1 /(1+n))$ and $\int_{\mathbb{R}^{3}} w^{n}=$ 1. These operators have the following well-known properties: there exists $C>0$ such that for all $f \in L^{2}\left(\mathbb{R}^{3}\right), r>1$ and $n \in \mathbb{N}$,

$$
\begin{gathered}
\left\|f-R^{n} f\right\|_{L^{2}\left(\mathbb{R}^{3}\right)} \rightarrow 0, \quad\left\|R^{n} f\right\|_{L^{2}\left(\mathbb{R}^{3}\right)} \leqslant C\|f\|_{L^{2}\left(\mathbb{R}^{3}\right)}, \\
\left\|R^{n} f\right\|_{L^{2}\left(B_{r}\right)} \leqslant C\|f\|_{L^{2}\left(B_{r+1}\right)}, \text { and }\left\|R^{n} f\right\|_{L^{2}\left(\mathbb{R}^{3} \backslash B_{r}\right)} \leqslant C\|f\|_{L^{2}\left(\mathbb{R}^{3} \backslash B_{r-1}\right)} .
\end{gathered}
$$

Moreover for all $n \in \mathbb{N}$, there exists $C_{n}>0$ such that for all $f \in L^{2}\left(\mathbb{R}^{3}\right)$,

$$
\left\|R^{n} f\right\|_{L^{\infty}\left(\mathbb{R}^{3}\right)} \leqslant C_{n}\|f\|_{L^{2}\left(\mathbb{R}^{3}\right)} .
$$

\subsection{Approximate solutions}

The following lemma claims the existence of global solutions to some regularized problem.

Lemma 3.1. For all $n \in \mathbb{N}$, there exists $U^{n}:=\left(u^{n}, v^{n}\right) \in C\left([0, \infty)\right.$, $\left.\mathbf{L}^{2}\right)$, with

$$
v^{n} \in C\left([0, \infty), L^{\infty}\left(\Omega, \mathbb{R}^{d}\right)\right) \cap C^{1}\left([0, \infty), L^{2}\left(\Omega, \mathbb{R}^{d}\right)\right),
$$

solution to the regularized problem:

$$
\begin{aligned}
\left(\partial_{t}+B\right) u^{n} & =\left(\kappa^{-1} \cdot l\right) F^{n} & & \text { for } x \in \mathbb{R}^{3}, \\
\partial_{t} v^{n} & =F^{n} & & \text { for } x \in \Omega,
\end{aligned}
$$

where

$$
F^{n}(t, x):=F\left(x, \overline{v^{n}}(t, x), R^{n} u^{n}(t, x)\right),
$$

with $U_{\text {init }}$ as initial data. Moreover, for all $n \in \mathbb{N}$,

(a) For almost all $x \in \mathbb{R}^{3}$, for all $t \geqslant 0,\left|v^{n}(t, x)\right| \leqslant\left|v_{\text {init }}(x)\right| e^{K t}$ (with $K$ from (2.5)).

(b) For all $T>0$, there is $C=C\left(T, F, l, \kappa,\left\|v_{\text {init }}\right\|_{L^{\infty}}\right)$ such that, for all $t \in$ $[0, T],\left\|\left(u^{n}, v^{n}\right)(t)\right\|_{\mathbf{L}^{2}}+\left\|\partial_{t} v^{n}(t)\right\|_{L^{2}(\Omega)} \leqslant C\left\|U_{\text {init }}\right\|_{\mathbf{L}^{2}}$.

Proof. The local-in-time solution is constructed via a usual fixed point argument for the mapping $\mathcal{A}^{n}: C\left([0, T], \mathbf{L}^{2}\right) \rightarrow C\left([0, T], \mathbf{L}^{2}\right)$,

$$
\begin{aligned}
& \mathcal{A}^{n}(u, v)(t, \cdot) \\
& =\left(\exp (-t B) u_{\text {init }}+\int_{0}^{t} \exp \left(\left(t-t^{\prime}\right) B\right)\left(\kappa^{-1} \cdot l\right) \mathcal{F}^{n}\left(t^{\prime}, \cdot\right) d t^{\prime}, v_{\text {init }}+\int_{0}^{t} \mathcal{F}^{n}\left(t^{\prime}, \cdot\right) d t^{\prime}\right),
\end{aligned}
$$

where

$$
\mathcal{F}^{n}(t, \cdot):=F\left(\cdot, \bar{v}(t, \cdot), R^{n} u(t, \cdot)\right) .
$$


For $T>0$ small enough, $\mathcal{A}^{n}$ is shown to be a contraction mapping thanks to properties (2.3)-(2.5) of $F,(3.4)$, and because $B$ is a skew self-adjoint operator in the Hilbert space $L^{2}\left(\mathbb{R}^{3}, \mathbb{R}^{6}\right)$ endowed with the scalar product $\langle\cdot, \cdot\rangle_{\kappa_{1}, \kappa_{2}}$.

Global existence is given by the a priori bounds (a) and (b). The first one follows directly from (2.5) and Gronwall's lemma. In the same way, taking the $L^{2}$ norm of $\left(u^{n}, v^{n}\right)(t)=\mathcal{A}^{n}\left(u^{n}, v^{n}\right)(t)$, one gets

$$
\left\|\left(u^{n}, v^{n}\right)(t)\right\|_{\mathbf{L}^{2}} \leqslant\left\|U_{\text {init }}\right\|_{\mathbf{L}^{2}}+\int_{0}^{t}\left(1+\left\|\kappa^{-1} \cdot l\right\|_{L^{\infty}}\right)\left\|F^{n}\left(t^{\prime}, \cdot\right)\right\|_{L^{2}} d t^{\prime} .
$$

One may add to this inequality the one obtained from (3.7),

$$
\left\|\partial_{t} v^{n}(t, \cdot)\right\|_{L^{2}} \leqslant\left\|F^{n}(t, \cdot)\right\|_{L^{2}} .
$$

From (2.3), (2.4), we have

$$
\left\|F^{n}(t, \cdot)\right\|_{L^{2}} \leqslant C_{F}\left(\left\|v_{\text {init }}\right\|_{\left.L^{\infty} e^{K t}\right)\left\|\left(u^{n}, v^{n}\right)(t)\right\|_{\mathbf{L}^{2}},}\right.
$$

so that Gronwall's lemma concludes.

\subsection{Passing to the limit $n \rightarrow \infty$}

Let us stress that Estimate (3.4) is not uniform with respect to $n$. However, we have:

Proposition 3.2. For all $T>0$, there is a subsequence of $\left(U^{n}\right)_{n \in \mathbb{N}}$ given by Lemma 3.1 that strongly converges in $C\left([0, T], \mathbf{L}^{2}\right)$ to $U:=(u, v) \in C\left([0, \infty), \mathbf{L}^{2}\right)$, global finite energy solution to (2.6)-(2.8) with $U_{\text {init }}$ as initial data, and satisfying the estimates (i), (ii), (iii) of Theorem 2.6.

Proof. First we infer from the bounds (a)-(b) in Lemma 3.1 that there exists a subsequence, still denoted $\left(u^{n}, v^{n}\right)$, such that $u^{n}$ (respectively $F^{n}$ ) tends to $u$ (respectively to $\left.F_{\text {lim }}\right)$ in $L^{\infty}\left((0, T), \mathbf{L}^{2}\right)$ weak $*\left(\right.$ respectively $L^{\infty}\left((0, T), L^{2}\left(\mathbb{R}^{3}\right)\right)$ weak $\left.*\right)$ and $v^{n}$ tends to $v$ in $W^{1, \infty}\left((0, T), L^{2}(\Omega)\right)$ weak $*$ and in $L^{\infty}\left((0, T), L^{\infty}(\Omega)\right)$ weak*. This is enough to ensure that $(u, v)$ satisfies (2.6). Moreover, Fatou's lemma yields that $u$ and $v$ satisfy (i)-(ii) of Theorem 2.6 for almost every $t$ in $(0, T)$.

Since the function $F$ is not linear, these weak limits do not suffice to pass to the limit in Equation (3.7). The strategy is to carefully study the nonlinear term $F^{n}$ to prove that the solutions $U^{n}$ of the regularized problems (3.6)-(3.7) actually converge (strongly) in $L^{2}$. The key step consists in proving the strong convergence of $v^{n}$.

It shall be useful several times to keep in mind that, thanks to the growth conditions (2.4) on $F$ and to the pointwise bound Lemma 3.1, (a) of the $v^{n}$, there holds, for all $n, m \in \mathbb{N}$, for all $(t, x) \in[0, T] \times \mathbb{R}^{3}$,

$$
\begin{aligned}
\left|F_{i}^{n}(t, x)\right| & \leqslant C_{F}\left(e^{K T}\left\|v_{\text {init }}\right\|_{L^{\infty}}\right), \\
\left|F_{i}^{n}(t, x)-F_{i}^{m}(t, x)\right| & \leqslant C_{F}\left(e^{K T}\left\|v_{\text {init }}\right\|_{L^{\infty}}\right)\left|\overline{v^{n}}(t, x)-\overline{v^{m}}(t, x)\right|,
\end{aligned}
$$

where $F_{i}^{n}=F_{i}\left(x, \overline{v^{n}}\right), i=0,1$. 
Strong convergence of $v^{n}$. We perform energy estimates on (3.6)-(3.7). Since $u$ may be unbounded, we introduce a weight function, which precisely depends on $u$. More exactly, we choose a positive function $\rho_{0}(x)$ in $L^{\infty}\left(\mathbb{R}^{3}\right) \cap L^{2}\left(\mathbb{R}^{3}\right)$ and define

$$
\rho(t, x):=\rho_{0}(x) e^{-L \int_{0}^{t}|u(s, x)| d s},
$$

with $L \geqslant C_{F}\left(e^{K T}\left\|v_{\text {init }}\right\|_{L^{\infty}}\right)$. First, using (3.7) we get

$$
\begin{aligned}
& \frac{1}{2} \frac{\mathrm{d}}{\mathrm{dt}}\left(\left\|\rho\left(v^{n}-v^{m}\right)\right\|_{L^{2}(\Omega)}^{2}\right)(t) \\
& =\int_{\Omega} \rho^{2}\left(v^{n}-v^{m}\right) \cdot\left(F^{n}-F^{m}\right) d x-L \int_{\Omega} \rho^{2}\left|u \| v^{n}-v^{m}\right|^{2} d x .
\end{aligned}
$$

Next, decompose $F^{n}-F^{m}$ according to (2.3) to get

$$
\begin{aligned}
& \frac{1}{2} \frac{\mathrm{d}}{\mathrm{dt}}\left(\left\|\rho\left(v^{n}-v^{m}\right)\right\|_{L^{2}(\Omega)}^{2}\right)(t)=\int_{\Omega} \rho^{2}\left(v^{n}-v^{m}\right) \cdot\left(F_{0}^{n}-F_{0}^{m}\right) d x \\
& \quad+\int_{\Omega} \rho^{2}\left(v^{n}-v^{m}\right) \cdot\left(F_{1}^{n} R^{n} u^{n}-F_{1}^{m} R^{m} u^{m}\right) d x-L \int_{\Omega} \rho^{2}|u|\left|v^{n}-v^{m}\right|^{2} d x .
\end{aligned}
$$

The first term in the right hand side of (3.12) can be estimated by $C \| \rho\left(v^{n}-\right.$ $\left.v^{m}\right) \|_{L^{2}(\Omega)}^{2}(t)$ thanks to (3.10). Now, decompose $F_{1}^{n} R^{n} u^{n}-F_{1}^{m} R^{m} u^{m}$ into

$$
F_{1}^{n} R^{n} u^{n}-F_{1}^{m} R^{m} u^{m}=F_{1}^{n}\left(R^{n} u^{n}-u\right)-F_{1}^{m}\left(R^{m} u^{m}-u\right)+\left(F_{1}^{n}-F_{1}^{m}\right) u .
$$

The terms produced by the third parenthesis are estimated thanks to (3.10), and absorbed by the last term in (3.12), so that

$$
\begin{aligned}
\frac{1}{2} \frac{\mathrm{d}}{\mathrm{dt}}\left(\left\|\rho\left(v^{n}-v^{m}\right)\right\|_{L^{2}(\Omega)}^{2}\right)(t) \leqslant & C\left\|\rho\left(v^{n}-v^{m}\right)\right\|_{L^{2}(\Omega)}^{2}(t) \\
& +\int_{\Omega} \rho^{2}\left(v^{n}-v^{m}\right) \cdot F_{1}^{n}\left(R^{n} u^{n}-u\right) d x \\
& +\int_{\Omega} \rho^{2}\left(v^{m}-v^{n}\right) \cdot F_{1}^{m}\left(R^{m} u^{m}-u\right) d x .
\end{aligned}
$$

Then, decompose $R^{n} u^{n}$ and $R^{m} u^{m}$ according to the orthogonal projector $P$ to get

$$
\begin{aligned}
\frac{1}{2} \frac{\mathrm{d}}{\mathrm{dt}}\left(\left\|\rho\left(v^{n}-v^{m}\right)\right\|_{L^{2}(\Omega)}^{2}\right)(t) \leqslant & C\left\|\rho\left(v^{n}-v^{m}\right)\right\|_{L^{2}(\Omega)}^{2}(t) \\
& +\sum_{j=1}^{3} h_{j, m, n}(t)+h_{j, n, m}(t),
\end{aligned}
$$


where

$$
\begin{aligned}
& h_{1, m, n}(t):=\int_{\Omega} \rho^{2}\left(v^{n}-v^{m}\right) \cdot F_{1}^{n} R^{n} P\left(u^{n}-u\right) d x, \\
& h_{2, m, n}(t):=\int_{\Omega} \rho^{2}\left(v^{n}-v^{m}\right) \cdot F_{1}^{n} R^{n}(I d-P)\left(u^{n}-u\right) d x, \\
& h_{3, m, n}(t):=\int_{\Omega} \rho^{2}\left(v^{n}-v^{m}\right) \cdot F_{1}^{n}\left(R^{n} u-u\right) d x .
\end{aligned}
$$

The following lemma deals with the term $h_{1, m, n}(t)$.

Lemma 3.3. There holds

$$
\forall \delta>0, \exists N_{\delta} \in \mathbb{N}, \forall n \geqslant N_{\delta}, \forall m \in \mathbb{N}, \quad\left|\int_{0}^{T} h_{1, m, n}(t) d t\right| \leqslant 2 \delta .
$$

Proof. First notice that

$$
h_{1, m, n}(t)=\int_{\mathbb{R}^{3}} R^{n}\left(\rho^{2}\left(\overline{v^{n}}-\overline{v^{m}}\right) \cdot F_{1}^{n}\right) P\left(u^{n}-u\right) d x .
$$

We first handle the case where $x$ is outside of a large ball. Using the CauchySchwarz inequality, the second property of $R^{n}$ in (3.3), the uniform bound in $L^{\infty}\left([0, T], L^{2}\left(\mathbb{R}^{3}\right)\right)$ for $v^{n}$ given in Lemma 3.1, (b) and the bound (3.9) for $F_{1}^{n}$, we get that

$$
\begin{aligned}
& \int_{0}^{T} \int_{\mathbb{R}^{3} \backslash B_{r}}\left|R^{n}\left(\rho^{2}\left(\overline{v^{n}}-\overline{v^{m}}\right) F_{1}^{n}\right) \cdot P\left(u^{n}-u\right)\right| d x d t \\
& \leqslant C \int_{0}^{T}\left\|\rho(t)^{2}\right\|_{L^{2}\left(\mathbb{R}^{3} \backslash B_{r-1}\right)} d t .
\end{aligned}
$$

By definition of $\rho$ there exists $r>0$ so that this integral is less than $\delta$. lemma:

It remains to tackle the case where $x \in B_{r}$. We use the following compactness

Lemma 3.4 (Jochmann [23, Lemma 3.4]). Let $\left(G^{n}\right)_{n \in \mathbb{N}}$ and $\left(K^{n}\right)_{n \in \mathbb{N}}$ be bounded sequences in $L^{\infty}\left([0, T), L^{2}\left(\mathbb{R}^{3}, \mathbb{R}^{6}\right)\right)$, with $K^{n}$ converging to 0 in $L^{\infty}([0, T)$, $L^{2}\left(\mathbb{R}^{3}, \mathbb{R}^{6}\right)$ ) weak $*$. Suppose that $\left(G^{n}\right)_{n \in \mathbb{N}}$ is equicontinuous from $[0, T]$ to $L^{2}\left(\mathbb{R}^{3}, \mathbb{R}^{6}\right)$ and that $B K^{n}=\partial_{t} C^{n}$ with $\left(C^{n}\right)_{n \in \mathbb{N}}$ bounded in $L^{\infty}\left([0, T), L^{2}\left(\mathbb{R}^{3}, \mathbb{R}^{6}\right)\right)$. Then for all $r>0$,

$$
\sup _{p \in \mathbb{N}}\left|\int_{0}^{T} \int_{B_{r}} G^{p}(t) \cdot P K^{n}(t) d x d t\right| \underset{n \rightarrow \infty}{\longrightarrow} 0
$$


Let us denote $G^{k, l}=R^{k}\left(\rho^{2}\left(\overline{v^{k}}-\overline{v^{l}}\right) \cdot F_{1}^{k}\right)$ and $K^{n}=u^{n}-u$. Thanks to (3.9), (3.2) and to Lemma 3.1, (b), we get that $\left(G^{k, l}\right)_{k, l \in \mathbb{N}}$ and $\left(K^{n}\right)_{n \in \mathbb{N}}$ are bounded in $L^{\infty}\left([0, T), L^{2}\left(\mathbb{R}^{3}\right)\right)$. Moreover $K^{n}$ tends to zero in $L^{\infty}\left([0, T), L^{2}\left(\mathbb{R}^{3}\right)\right)$ weak $*$, by definition of $u$. Let us denote $\mathcal{F}^{n}=\int_{0}^{t}\left(\kappa^{-1} \cdot l\right) F^{n} d t^{\prime}$ and $\mathcal{F}=\int_{0}^{t}\left(\kappa^{-1} \cdot l\right) F_{\text {lim }} d t^{\prime}$. From (3.6) we infer that

$$
B K^{n}=\partial_{t} C^{n}, \quad \text { with } \quad C^{n}:=\mathcal{F}^{n}-u^{n}-(\mathcal{F}-u) .
$$

The sequence $\left(C^{n}\right)_{n \in \mathbb{N}}$ is bounded in $L^{\infty}\left([0, T), L^{2}\left(\mathbb{R}^{3}\right)\right)$. In the same way, equicontinuity is obtained from the bounds on $\partial_{t} v^{n}=F^{n}$. We therefore apply the lemma observing that, for all $m, n \in \mathbb{N}$,

$$
\left|\int_{0}^{T} \int_{B_{r}} G^{m, n}(t) \cdot P K^{n}(t) d x d t\right| \leqslant \sup _{k, l \in \mathbb{N}}\left|\int_{0}^{T} \int_{B_{r}} G^{k, l}(t) \cdot P K^{n}(t) d x d t\right| .
$$

Lemma 3.4 therefore ensures that there is $N_{r, \delta} \in \mathbb{N}$ such that, for $n \geqslant N_{r, \delta}$ and for all $m \in \mathbb{N}$,

$$
\left|\int_{0}^{T} \int_{B_{r}} R^{n}\left(\rho^{2}\left(\overline{v^{n}}-\overline{v^{m}}\right) F_{1}^{n}\right) \cdot P\left(u^{n}-u\right) d x d t\right| \leqslant \delta,
$$

and Lemma 3.3 is proved.

We now deal with the term $h_{2, m, n}(t)$.

Lemma 3.5. There holds

$$
\begin{aligned}
& \forall \delta>0, \exists N_{\delta} \in \mathbb{N}, \forall n \geqslant N_{\delta}, \forall m \in \mathbb{N}, \\
& \left|\int_{0}^{T} h_{2, m, n}(t) d t\right| \leqslant \delta+C\left\|\rho\left(\overline{v^{n}}-\overline{v^{m}}\right)\right\|_{L_{t, x}^{2}}\left\|\rho\left(\overline{v^{n}}-\bar{v}\right)\right\|_{L_{t, x}^{2}} .
\end{aligned}
$$

Proof. The $\left(u^{n}, v^{n}\right)$ satisfy (2.6) and so does their weak limit $(u, v)$. Thus

$$
\begin{aligned}
h_{2, m, n}(t) & =-\int_{\Omega} \rho^{2}\left(v^{n}-v^{m}\right) \cdot F_{1}^{n} R^{n}(I d-P)\left(\kappa^{-1} \cdot l\right)\left(\overline{v^{n}}-\bar{v}\right) d x \\
& =-\int_{\mathbb{R}^{3}} \rho^{2}\left(\overline{v^{n}}-\overline{v^{m}}\right) \cdot F_{1}^{n} R^{n}(I d-P)\left(\kappa^{-1} \cdot l\right)\left(\overline{v^{n}}-\bar{v}\right) d x .
\end{aligned}
$$

Then we decompose

$$
\begin{aligned}
& \int_{0}^{T}\left|h_{2, m, n}(t)\right| d t \leqslant \int_{0}^{T} \mid \int_{\mathbb{R}^{3}}\left(\overline{v^{n}}-\overline{v^{m}}\right) \cdot F_{1}^{n}\left[\rho^{2} R^{n}(I d-P)\left(\kappa^{-1} \cdot l\right)\left(\overline{v^{n}}-\bar{v}\right)\right. \\
& \left.\quad-\rho R^{n}(I d-P) \rho\left(\kappa^{-1} \cdot l\right)\left(\overline{v^{n}}-\bar{v}\right)\right] d x \mid d t \\
& \quad+\int_{0}^{T}\left|\int_{\mathbb{R}^{3}} \rho\left(\overline{v^{n}}-\overline{v^{m}}\right) \cdot F_{1}^{n} R^{n}(I d-P) \rho\left(\kappa^{-1} \cdot l\right)\left(\overline{v^{n}}-\bar{v}\right) d x\right| d t .
\end{aligned}
$$


The second integral in the right hand side of (3.18) is estimated thanks to Hölder's inequality:

$$
\begin{aligned}
& \int_{0}^{T}\left|\int_{\mathbb{R}^{3}} \rho\left(\overline{v^{n}}-\overline{v^{m}}\right) \cdot F_{1}^{n} R^{n}(I d-P) \rho\left(\kappa^{-1} \cdot l\right)\left(\overline{v^{n}}-\bar{v}\right) d x\right| d t \\
& \leqslant C\left\|\rho\left(\overline{v^{n}}-\overline{v^{m}}\right)\right\|_{L_{t, x}^{2}}\left\|\rho\left(\overline{v^{n}}-\bar{v}\right)\right\|_{L_{t, x}^{2}},
\end{aligned}
$$

where $C$ depends only on $T, F, l,\left\|v_{\text {init }}\right\|_{L^{\infty}}$. To deal with the first integral in the right-hand side of (3.18), we use the following commutation lemma.

Lemma 3.6 (Jochmann [23, Lemma 3.5]). Let $\rho$ belong to $L^{2}\left((0, T), L^{2}\left(\mathbb{R}^{3}\right)\right) \cap$ $L^{\infty}\left((0, T), L^{\infty}\left(\mathbb{R}^{3}\right)\right)$, and let $\left(M^{n}\right)_{n \in \mathbb{N}}$ be a bounded sequence in $W^{1, \infty}((0, T)$, $\left.L^{2}\left(\mathbb{R}^{3}\right)\right) \cap L^{\infty}\left((0, T), L^{\infty}\left(\mathbb{R}^{3}\right)\right)$ which converges to 0 in $L^{\infty}\left((0, T), L^{2}\left(\mathbb{R}^{3}\right)\right)$ weak $*$. Then

$$
\begin{aligned}
& \int_{0}^{T}\left\|\rho(t)^{2} R^{n}(I d-P) M^{n}-\rho(t) R^{n}(I d-P) \rho(t) M^{n}\right\|_{L^{1}\left(\mathbb{R}^{3}\right)+L^{2}\left(\mathbb{R}^{3}\right)} d t \underset{n \rightarrow \infty}{\longrightarrow} 0 \\
& \text { and } \int_{0}^{T}\left\|\rho(t)^{2}(I d-P) M^{n}-\rho(t)(I d-P) \rho(t) M^{n}\right\|_{L^{1}\left(\mathbb{R}^{3}\right)+L^{2}\left(\mathbb{R}^{3}\right)} d t \underset{n \rightarrow \infty}{\longrightarrow} 0 .
\end{aligned}
$$

We apply Lemma 3.6, (3.19) with $M^{n}=\left(\kappa^{-1} \cdot l\right)\left(\overline{v^{n}}-\bar{v}\right)$ : the first integral in the right-hand side of (3.18) is estimated by

$C\left(T, F,\left\|v_{\text {init }}\right\|_{\left.L^{\infty}\right)} \int_{0}^{T}\left\|\overline{v^{n}}-\overline{v^{m}}\right\|_{L^{\infty} \cap L^{2}}\left\|\rho^{2} R^{n}(I d-P) M^{n}-\rho R^{n}(I d-P) \rho M^{n}\right\|_{L^{1}+L^{2}} d t\right.$,

and thus goes to zero as $n$ goes to infinity, uniformly with respect to $m$. Hence, we get (3.17).

For all $\delta>0$, we also bound $h_{3, m, n}$ by $\delta$ for all $n \geqslant N_{\delta}$ and all $m \in \mathbb{N}$ thanks to (3.2). Finally, summing up with (3.14) and (3.17), we have from (3.13):

$$
\begin{aligned}
& \forall \delta>0, \exists N_{\delta} \in \mathbb{N}, \forall n, m \geqslant N_{\delta}, \forall t \in[0, T], \\
& \left\|\rho\left(v^{n}-v^{m}\right)\right\|_{L^{2}(\Omega)}^{2}(t) \\
& \leqslant C\left(\delta+\left\|\rho\left(v^{n}-v^{m}\right)\right\|_{L^{2}((0, T) \times \Omega)}^{2}+\left\|\rho\left(v^{n}-v\right)\right\|_{L^{2}((0, T) \times \Omega)}^{2}\right) .
\end{aligned}
$$

Use Gronwall's Lemma, then let $m$ go to $\infty$, and use Gronwall's Lemma again to deduce:

$$
\forall \delta>0, \exists N_{\delta} \in \mathbb{N}, \forall n \geqslant N_{\delta}, \forall t \in[0, T], \quad\left\|\rho\left(v^{n}-v\right)\right\|_{L^{2}(\Omega)}^{2}(t) \leqslant C \delta,
$$

which implies that $v^{n}$ converges towards $v$ strongly in $L^{2}\left((0, T) \times \Omega, \rho(t, x)^{2} d t d x\right)$. Up to a subsequence, convergence then holds almost everywhere, for the measure $\rho^{2} d t d x$, or $d t d x$, since $\rho$ is positive almost everywhere in $(0, T) \times \Omega$. Thanks to the 
pointwise estimates (a) from Lemma 3.1, dominated convergence thus ensures that $v^{n}$ converges towards $v$ strongly in $L^{2}((0, T) \times \Omega, d t d x)$. Then, equicontinuity of $\{v\} \cup\left\{v^{n}\right\}_{n \in \mathbb{N}}$ in $C\left([0, T], L^{2}(\Omega)\right)$ implies (by Ascoli's Theorem) the strong convergence of $v^{n}$ in $C\left([0, T], L^{2}(\Omega)\right)$. This, together with the uniform bounds on $\{v\} \cup\left\{v^{n}\right\}_{n \in \mathbb{N}}$ and with the weak convergence of $u^{n}$, is enough to pass to the limit in (3.6), (3.7) to get (2.7), (2.8).

Strong convergence of $u^{n}$. Since $u^{n}$ and $u$ satisfy (3.6) and (2.7) respectively, their difference is solution to a hyperbolic equation with source term in $L^{1}\left((0, T), L^{2}\left(\mathbb{R}^{3}\right)\right)$,

$$
\left(\partial_{t}+B\right)\left(u^{n}-u\right)=\left(\kappa^{-1} \cdot l\right)\left(F^{n}-F(x, \bar{v}, u)\right) .
$$

The standard energy estimate then gives

$$
\begin{aligned}
& \left\|u^{n}-u\right\|_{L^{2}}(t) \leqslant C \int_{0}^{t}\left\|F^{n}-F(x, \bar{v}, u)\right\|_{L^{2}}\left(t^{\prime}\right) d t^{\prime} \\
& \leqslant C \int_{0}^{t}\left(\left\|F_{0}^{n}-F_{0}(x, \bar{v})\right\|_{L^{2}}\left(t^{\prime}\right)+\left\|F_{1}^{n} R^{n} u^{n}-F_{1}(x, \bar{v}) u\right\|_{L^{2}}\left(t^{\prime}\right)\right) d t^{\prime} .
\end{aligned}
$$

Thanks to the growth conditions (2.4) of $F$ and to the pointwise bound Lemma 3.1, (a) of the $v^{n}$, there holds, for all $n, m \in \mathbb{N}$ and $(t, x) \in[0, T] \times \mathbb{R}^{3}$,

$$
\left|F_{i}^{n}(t, x)-F_{i}(x, \bar{v}(t, x))\right| \leqslant C_{F}\left(e^{K T}\left\|v_{\text {init }}\right\|_{L^{\infty}}\right)\left|\overline{v^{n}}(t, x)-\bar{v}(t, x)\right| .
$$

In particular this yields that for any $t^{\prime} \in[0, T],\left\|F_{0}^{n}-F_{0}(x, \bar{v})\right\|_{L^{2}}\left(t^{\prime}\right)$ goes to zero as $n$ goes to infinity.

Furthermore,

$$
\left|F_{1}^{n} R^{n} u^{n}-F_{1}(x, \bar{v}) u\right| \leqslant\left|F_{1}^{n}\right|\left|R^{n}\left(u^{n}-u\right)\right|+\left|F_{1}^{n}\right|\left|\left(R^{n}-I d\right) u\right|+\left|F_{1}^{n}-F_{1}(x, \bar{v})\right||u| .
$$

Thanks to the $L^{\infty}$ bounds on $v^{n}$ (cf. Lemma 3.1, (a)) and on $F_{1}^{n}(c f$. (3.9)), and to the property (3.2) of the operator $R^{n}$, the first term in the right hand side above is bounded by $C_{F}\left(e^{K T}\left\|v_{\text {init }}\right\|_{L^{\infty}}\right)\left|u^{n}-u\right|$. In the same way, the second term goes to zero in $L^{2}$ as $n$ goes to infinity. Finally, up to a subsequence, the third term tends to zero almost everywhere, and is bounded by $C\left(F, T,\left\|v_{\text {init }}\right\|_{L^{\infty}}\right)|u|$. By dominated convergence, it thus goes to zero in $L^{2}$. Finally, we get from (3.22):

$$
\left\|u^{n}-u\right\|_{L^{2}}(t) \leqslant C\left(F, T,\left\|v_{\text {init }}\right\|_{L^{\infty}}\right) \int_{0}^{t}\left\|u^{n}-u\right\|_{L^{2}}\left(t^{\prime}\right) d t^{\prime}+o(1),
$$

and Gronwall's Lemma shows that $u^{n}$ converges to $u$ in $C\left([0, T], L^{2}\right)$.

Thanks to a diagonal extraction process (using times $T \in \mathbb{N}^{\star}$ ), Proposition 3.2 produces $U \in C\left([0, \infty), L^{2}\right)$, solution to (2.6)-(2.8) with $U_{\text {init }}$ as initial data. Estimates (i) and (ii) are then straightforward. To prove Theorem 2.6, there remains to show its last statement: the stability property. To this end, consider a 
sequence $\left(U_{\text {init }}^{n}\right)_{n \in \mathbb{N}}$, bounded in $L_{\text {div }}$, and converging to $U_{\text {init }}$ in $L^{2}$. It generates a (sub)sequence of solutions $\left(U^{n}\right)_{n \in \mathbb{N}}$, with, from the bounds (i), (ii) in Theorem 2.6, $u^{n}$ converging to $u$ in $L_{\mathrm{loc}}^{\infty}\left((0, \infty), L^{2}\left(\mathbb{R}^{3}\right)\right)$ weak $*$ and $v^{n}$ converging to $v$ in $W_{\text {loc }}^{1, \infty}\left((0, \infty), L^{2}(\Omega)\right)$ weakly $*$ and in $L_{\text {loc }}^{\infty}\left((0, \infty), L^{\infty}(\Omega)\right)$ weak $*$. Then, define the weight $\rho$ from (3.11) and estimate $v^{n}-v^{m}$ as in (3.12), with $R^{n} u^{n}$ and $R^{m} u^{m}$ replaced with $u^{n}$ and $u^{m}$, respectively. This leads to the analogue to (3.13), with no $h_{3, m, n}$ and $h_{3, n, m}$ terms, and no $R^{n}$ in $h_{1, m, n}$ and $h_{2, m, n}$. Apply Lemma 3.4 and Lemma 3.6, (3.20) (instead of (3.19)), to get strong $C\left([0, T], L^{2}\right)$ convergence of $v^{n}$ towards $v$ (in (3.21), the term $\left\|v_{\text {init }}^{n}-v_{\text {init }}^{m}\right\|_{L^{2}}$ goes to zero, and contributes to $\delta)$. Strong convergence of the fields $u^{n}$ is then obtained as above, with an initial term $\left\|u_{\text {init }}^{n}-u_{\text {init }}\right\|_{L^{2}}$ going to zero added to the right hand side of (3.22).

The same process proves Proposition 2.7.

\section{Propagation of smoothness and uniqueness: proof of Theorem 2.8}

It is worth noting that, under the smoothness assumption on $\varepsilon$ and $\mu$ in (2.11), $u \in L^{2}\left(\mathbb{R}^{3}, \mathbb{R}^{6}\right)$ with $P u \in H^{\mu}\left(\mathbb{R}^{3}, \mathbb{R}^{6}\right)$ iff $u \in L^{2}\left(\mathbb{R}^{3}, \mathbb{R}^{6}\right)$ with curl $u_{i} \in$ $H^{\mu-1}\left(\mathbb{R}^{3}, \mathbb{R}^{6}\right)$ for $i=1,2$.

We thus split the proof of Theorem 2.8 in several steps. In Section 4.1, we isolate a Cauchy problem for the projection $P u$ of $u$. This allows some dispersive estimates that we etablish in Section 4.3, while in Section 4.2, Littlewood-Paley decompositions are introduced. We consider first the case where $\mu$ is in $(0,1)$, then we prove the part (a) of Theorem 2.8 in the case $\mu=1$, which concerns the propagation of smoothness, and finally the part (b), which concerns uniqueness.

Remark 4.1. Let us mention that in the proof of the propagation of $H^{1}$ regularity given in [18], the step " $\mu \in(0,1)$ " is missing, and the resulting estimates (collected here in Lemma 4.18) are claimed without proof.

\subsection{Preliminaries}

Lemma 4.2. For any solution $U:=(u, v)$ to (2.6)-(2.8) with $U_{\text {init }}:=\left(u_{\text {init }}, v_{\text {init }}\right) \in$ $L_{\mathrm{div}}$ as initial data given by Theorem 2.6 , the part $\mathbf{u}:=$ Pu solves for $x \in \mathbb{R}^{3}$,

$$
\begin{aligned}
\left(\partial_{t}+B\right) \mathbf{u} & =P(A \mathbf{u})+P g, \\
\left.\mathbf{u}\right|_{t=0} & =P u_{\text {init }},
\end{aligned}
$$

where

$$
\begin{aligned}
A(t, x) & :=\left(\kappa^{-1} \cdot l\right) F_{1}(x, \bar{v}) \\
g(t, x) & :=\left(\kappa^{-1} \cdot l\right) F\left(x, \bar{v},(I d-P)\left(\kappa^{-1} \cdot l\right) \bar{v}\right), \\
& \left.=\left(\kappa^{-1} \cdot l\right) F_{0}(x, \bar{v})+\left(\kappa^{-1} \cdot l\right) F_{1}(x, \bar{v})(I d-P)\left(\kappa^{-1} \cdot l\right) \bar{v}\right) .
\end{aligned}
$$


Proof. First, apply the projector $P$ to the system (2.7), observing that $P$ commutes with both $\partial_{t}$ and $B$. Then, split $F$ according to (2.3), split $u$ into $u=\mathbf{u}+(I d-P) u$ and finally use the constraint (2.6).

The projectors $P_{i}, i=1,2$, defined on $L^{2}\left(\mathbb{R}^{3}, \mathbb{R}^{3}\right)$, extend to $L^{p}\left(\mathbb{R}^{3}, \mathbb{R}^{3}\right)$ (this result extends the classical one by Calderón and Zygmund [11] on singular integrals, in the spirit of the extension by Judovič [46]):

Lemma 4.3 (Starynkevitch [43, Lemma 3.13]). Under assumption (2.11), the projectors $P_{i}, i=1,2$, extend to $L^{p}\left(\mathbb{R}^{3}, \mathbb{R}^{3}\right)$ and for all $p_{0}>1$, there exists $C>0$ such that for all $p \in\left[p_{0}, \infty\right)$, their norm from $L^{p}\left(\mathbb{R}^{3}, \mathbb{R}^{3}\right)$ into itself are less than $C p$.

We deduce estimates for the right-hand side of (4.1):

Lemma 4.4. As in Theorem 2.8, assume (2.3)-(2.5) and (2.11). Let $U_{\text {init }}:=$ $\left(u_{\text {init }}, v_{\text {init }}\right) \in L_{\mathrm{div}}$, and let $U:=(u, v)$ be any solution to $(2.6)-(2.8)$ with $U_{\text {init }}:=$ $\left(u_{\text {init }}, v_{\text {init }}\right) \in L_{\text {div }}$ as initial data given by Theorem 2.6. The following holds true for $A$ and $g$ given by (4.3)-(4.5):

$$
\begin{aligned}
A & \in L_{\mathrm{loc}}^{\infty}\left((0, \infty), L^{\infty}\left(\mathbb{R}^{3}\right)\right), \\
A & \in C\left([0, \infty), L^{2}\left(\mathbb{R}^{3}\right)\right), \\
\partial_{t} A & \in L_{\mathrm{loc}}^{\infty}\left((0, \infty), L^{2}\left(\mathbb{R}^{3}\right)\right), \\
g & \in \cap_{1 \leqslant p<\infty} C\left([0, \infty), L^{p}\left(\mathbb{R}^{3}\right)\right), \\
\partial_{t} g & \in \cap_{1 \leqslant q \leqslant 2} L_{\mathrm{loc}}^{\infty}\left((0, \infty), L^{p}\left(\mathbb{R}^{3}\right)\right) .
\end{aligned}
$$

Proof. For all $t, t^{\prime} \geqslant 0$, there holds

$$
\begin{gathered}
\|A(t)\|_{L^{\infty}\left(\mathbb{R}^{3}\right)} \leqslant\left\|\kappa^{-1} \cdot l\right\|_{L^{\infty}\left(\mathbb{R}^{3}\right)} C_{F}\left(\left\|v_{\text {init }}\right\|_{L^{\infty}} e^{K t}\right), \\
\left\|A(t)-A\left(t^{\prime}\right)\right\|_{L^{2}\left(\mathbb{R}^{3}\right)} \leqslant\left\|\kappa^{-1} \cdot l\right\|_{L^{\infty}\left(\mathbb{R}^{3}\right)} C_{F}\left(\left\|v_{\text {init }}\right\|_{\left.L^{\infty} e^{K t}\right)\left\|v(t)-v\left(t^{\prime}\right)\right\|_{L^{2}(\Omega)},}\right. \\
\left\|\partial_{t} A(t)\right\|_{L^{2}\left(\mathbb{R}^{3}\right)} \leqslant\left\|\kappa^{-1} \cdot l\right\|_{L^{\infty}\left(\mathbb{R}^{3}\right)} C_{F}\left(\left\|v_{\text {init }}\right\|_{L^{\infty}} e^{K t}\right)\left\|\partial_{t} v(t)\right\|_{L^{2}(\Omega)},
\end{gathered}
$$

what yields estimates (4.6)-(4.8).

Since $v \in C\left([0, \infty), L^{2}(\Omega)\right) \cap L_{\mathrm{loc}}^{\infty}\left((0, \infty), L^{\infty}(\Omega)\right)$, we have $v \in \cap_{p \geqslant 1} C([0, \infty)$, $L^{p}(\Omega)$ ) - using the boundedness of $\Omega$ for $p<2$, and by interpolation for $p>2$. Lemma 4.3 then yields (4.9). Next, using that $\left|F_{i}(x, v)\right| \leqslant C_{F}\left(\left\|v_{\text {init }}\right\|_{L^{\infty}} e^{K t}\right)|v|$ for $i=0$, 1 , we infer from (2.8) that $\partial_{t} v \in \cap_{1 \leqslant q \leqslant 2} L_{\text {loc }}^{\infty}\left((0, \infty), L^{p}\left(\mathbb{R}^{3}\right)\right)$. Since

$$
\begin{aligned}
\partial_{t} g(t, x)= & \left(\kappa^{-1} \cdot l\right)\left\{\partial_{v} F_{0}(x, \bar{v}) \cdot \partial_{t} \bar{v}+F_{1}(x, \bar{v})(I d-P)\left(\kappa^{-1} \cdot l\right)\right. \\
& \left.\cdot \partial_{t} \bar{v}+\left(\partial_{v} F_{1}(x, \bar{v}) \cdot \partial_{t} \bar{v}\right) \cdot(I d-P)\left(\kappa^{-1} \cdot l\right) \bar{v}\right\},
\end{aligned}
$$

thanks to Lemma 4.3, we finally get (4.10).

Also, for a given $v$, the "fields part" $u$ is in fact uniquely determined: 
Lemma 4.5. Let $A \in L_{\mathrm{loc}}^{\infty}\left((0, \infty), L^{\infty}\left(\mathbb{R}^{3}\right)\right)$ and $g \in L_{\mathrm{loc}}^{1}\left((0, \infty), L^{2}\left(\mathbb{R}^{3}\right)\right)$. For any $u_{\text {init }} \in L^{2}\left(\mathbb{R}^{3}\right)$, there exists only one solution $\mathbf{u} \in C\left([0, \infty), L^{2}\left(\mathbb{R}^{3}\right)\right)$ to (4.1)(4.2) with $P u_{\text {init }}$ as initial data. Furthermore, it satisfies $\mathbf{u}=P \mathbf{u}$.

Proof. Existence is given by Lemma 4.2. To prove uniqueness, consider two solutions $\mathbf{u}_{1}$ and $\mathbf{u}_{2}$ in $C\left([0, \infty), L^{2}\left(\mathbb{R}^{3}\right)\right)$ to (4.1)-(4.2), and $T>0$. Then

$$
\forall t \geqslant 0, \quad\left(\mathbf{u}_{1}-\mathbf{u}_{2}\right)(t)=\int_{0}^{t} e^{i(t-s) B} P A\left(\mathbf{u}_{1}-\mathbf{u}_{2}\right)(s) d s,
$$

so that, using (4.6), for $t \in[0, T]$,

$$
\left\|\left(\mathbf{u}_{1}-\mathbf{u}_{2}\right)(t)\right\|_{L^{2}\left(\mathbb{R}^{3}\right)} \leqslant C(\kappa, T) \int_{0}^{t}\left\|\left(\mathbf{u}_{1}-\mathbf{u}_{2}\right)(s)\right\|_{L^{2}\left(\mathbb{R}^{3}\right)} d s .
$$

Hence, by Gronwall's Lemma, $\mathbf{u}_{1}=\mathbf{u}_{2}$ on [0,T], for any $T>0$. Thus, there is only one solution $\mathbf{u}$. Finally, in the same way, $\mathbf{u}-P \mathbf{u}$ simply satisfies:

$$
\forall t \geqslant 0, \quad\left(\partial_{t}+B\right)(\mathbf{u}-P \mathbf{u})(t)=0,
$$

so that $\mathbf{u}=P \mathbf{u}$.

\subsection{Technical interlude 2: Fourier analysis}

We recall the existence of a smooth dyadic partition of unity: there exist two radial bump functions $\chi$ and $\phi$ valued in the interval [0,1], supported respectively in the ball $B(0,4 / 3):=\{|\xi|<4 / 3\}$ and in the annulus $C(3 / 4,8 / 3):=\{3 / 4<|\xi|<$ $8 / 3\}$, such that

$$
\begin{aligned}
& \forall \xi \in \mathbb{R}^{3}, \quad \chi(\xi)+\sum_{j \geqslant 0} \phi\left(2^{-j} \xi\right)=1, \quad \forall \xi \in \mathbb{R}^{3} \backslash\{0\}, \quad \sum_{j \in \mathbb{Z}} \phi\left(2^{-j} \xi\right)=1, \\
& \left|j-j^{\prime}\right| \geqslant 2 \Rightarrow \operatorname{supp} \phi\left(2^{-j} \cdot\right) \cap \operatorname{supp} \phi\left(2^{-j^{\prime}} .\right)=\emptyset, \\
& j \geqslant 1 \Rightarrow \operatorname{supp} \chi\left(2^{-j} \cdot\right) \cap \operatorname{supp} \phi\left(2^{-j} \cdot\right)=\emptyset .
\end{aligned}
$$

The Fourier transform $\mathcal{F}$ is defined on the space of integrable functions $f \in L^{1}\left(\mathbb{R}^{3}\right)$ by $(\mathcal{F} f)(\xi):=\int_{\mathbb{R}^{3}} e^{-2 i \pi x \cdot \xi} f(x) d x$, and extended to an automorphism of the space $\mathcal{S}^{\prime}\left(\mathbb{R}^{3}\right)$ of tempered distributions, which is the dual of the Schwartz space $\mathcal{S}\left(\mathbb{R}^{3}\right)$ of rapidly decreasing functions.

The so-called dyadic blocks $\Delta_{j}$ correspond to the Fourier multipliers $\Delta_{j}:=$ $\phi\left(2^{-j} D\right)$, that is

$$
\Delta_{j} u(x):=2^{3 j} \int_{\mathbb{R}^{3}} \tilde{h}\left(2^{j} y\right) u(x-y) d y \quad \text { for } j \geqslant 0, \quad \text { where } \tilde{h}:=\mathcal{F}^{-1} \phi .
$$


We also introduce $S_{0}:=\chi(D)$, that is

$$
S_{0} u(x):=\int_{\mathbb{R}^{3}} h(y) u(x-y) d y, \quad \text { where } h:=\mathcal{F}^{-1} \chi .
$$

We will use the inhomogeneous Littlewood-Paley decomposition $I d=S_{-1}+$ $\sum_{j \in \mathbb{N}} \Delta_{j}$, which holds in the space of tempered distributions $\mathcal{S}^{\prime}\left(\mathbb{R}^{3}\right)$, and the homogeneous Littlewood-Paley decomposition $I d=\sum_{j \in \mathbb{Z}} \Delta_{j}$, which holds in $\mathcal{S}_{h}^{\prime}\left(\mathbb{R}^{3}\right)$, the space of tempered distributions $u$ such that $\lim _{j \rightarrow-\infty}\left\|\sum_{k \leqslant j} \Delta_{k} u\right\|_{L^{\infty}\left(\mathbb{R}^{3}\right)}=0$.

We now recall the definition of the inhomogeneous (respectively homogeneous) Besov spaces $B_{p, q}^{\lambda}$ (respectively $\dot{B}_{p, q}^{\lambda}$ ) on $\mathbb{R}^{3}$ which are, for $\lambda \in \mathbb{R}$ (the smoothness index), $p, q \in[1,+\infty]$ (respectively the integral-exponent and the sum-exponent), the spaces of tempered distributions $u$ in $\mathcal{S}^{\prime}\left(\mathbb{R}^{3}\right)$ (respectively $\mathcal{S}_{h}^{\prime}\left(\mathbb{R}^{3}\right)$ ) such that

$$
\begin{aligned}
&\|f\|_{B_{p, q}^{\lambda}\left(\mathbb{R}^{3}\right)}:=\left\|S_{0} f\right\|_{L^{p}\left(\mathbb{R}^{3}\right)}+\left\|\left(2^{j \lambda}\left\|\Delta_{j} f\right\|_{L^{p}\left(\mathbb{R}^{3}\right)}\right)_{j}\right\|_{l^{q}(\mathbb{N})} \\
&\text { (respectively } \left.\|f\|_{\dot{B}_{p, q}^{\lambda}\left(\mathbb{R}^{3}\right)}:=\left\|\left(2^{j \lambda}\left\|\Delta_{j} f\right\|_{L^{p}\left(\mathbb{R}^{3}\right)}\right)_{j}\right\|_{l q}(\mathbb{Z})\right)
\end{aligned}
$$

is finite. These Banach spaces do not depend on the choice of the dyadic partition above ( $c f$. for instance the book [3]).

\subsection{Dispersion}

Propagation of smoothness or singularities for solutions to hyperbolic Cauchy problems, such as

$$
L u:=\left(\partial_{t}+B\right) u=f, \text { with }\left.u\right|_{t=0}=u_{\text {init }},
$$

obeys the laws of geometrical optics. Let us refer here to the survey [19] by Gårding for an introduction to the subject. The characteristic variety of the operator $L$ is defined as

$$
\operatorname{Char}(L):=\left\{(t, x, \tau, \xi) \in \mathbb{R} \times \mathbb{R}^{3} \times \mathbb{C} \times\left(\mathbb{R}^{3} \backslash\{0\}\right) \mid \operatorname{det} L(t, x, \tau, \xi)=0\right\},
$$

where $L(t, x, \tau, \xi)$ denotes the (principal) symbol of the operator $L$, which is the $6 \times 6$ matrix

$$
\begin{aligned}
L(t, x, \tau, \xi) \equiv L(x, \tau, \xi) & :=\tau I d+B(x, \xi), \\
\text { where } B(x, \xi) & :=\left[\begin{array}{cc}
0 & \kappa_{1}(x)^{-1} \xi \wedge \cdot \\
\kappa_{2}(x)^{-1} \xi \wedge \cdot & 0
\end{array}\right] .
\end{aligned}
$$

For all $(x, \xi) \in \mathbb{R}^{3} \times\left(\mathbb{R}^{3} \backslash\{0\}\right)$, the matrix $B(x, \xi)$ admits three eigenvalues: $\lambda_{ \pm}(x, \xi):= \pm\left(\kappa_{1} \kappa_{2}(x)\right)^{-1 / 2}|\xi|$ and 0 , each one with multiplicity 2 . The eigenspace associated with the eigenvalue 0 is precisely ran $(I d-P)$. We introduce

$$
P_{ \pm}(x, \xi):=\frac{1}{2 \pi i} \oint_{\left|z-\lambda_{ \pm}(x, \xi)\right|=r} L(x,-z, \xi)^{-1} d z
$$


where $r$ is chosen small enough for $\lambda_{ \pm}(x, \xi)$ being the only eigenvalue inside the circle of integration. The matrix $P_{ \pm}(x, \xi)$ is the spectral projection associated with the eigenvalue $\lambda_{ \pm}(x, \xi)$, that is the projection onto the kernel of $L\left(x,-\lambda_{ \pm}(x, \xi), \xi\right)$ along its range. These spectral projections are homogeneous of degree 0 with respect to $\xi$, and the associated pseudo-differential operators $P_{ \pm}$satisfy $P_{+}+P_{-}=$ $I d-P$. In addition, $I d-P, P_{+}$and $P_{-}$are orthogonal projectors (in the weighted $L^{2}$ space introduced in Section 2.1) commuting with $B$, and acting on Besov spaces.

Now the point is that considering the Cauchy problem (4.14) for solutions $u$ satisfying $(I d-P) u=0$, we select the branch of the characteristic variety which are curved, what generates dispersion. We shall need the following indices $p_{1}, r_{1}$, $q_{1}, s_{1}, \mu, \sigma$ and $\rho$ :

$$
\begin{aligned}
p_{1} & \in[2, \infty) \text { and } 1 / r_{1}+1 / p_{1}=1 / 2 ; \\
q_{1} & \in(1,2] \text { and } 1 / s_{1}+1 / q_{1}=3 / 2 ; \\
\mu & \in \mathbb{R}, \quad \sigma:=\mu-1+2 / p_{1} \text { and } \rho:=\mu-1+2 / q_{1} .
\end{aligned}
$$

Proposition 4.6. Let $p_{1}, r_{1}, q_{1}, s_{1}, \mu, \sigma$ and $\rho$ be given by (4.15)-(4.17). Under assumption (2.1), there is a non-decreasing function $C:(0, \infty) \rightarrow(0, \infty)$ such that, for any $T>0$, for any initial data $u_{\text {init }}$ in $\dot{H}^{\mu}$ such that $(I d-P) u_{\mathrm{init}}=0$, and for any source term $f$ in $L^{s_{1}}\left((0, T), \dot{B}_{q_{1}, 2}^{\rho}\left(\mathbb{R}^{3}\right)\right)$ such that $(I d-P) f=0$, any (weak) solution $u$ to the Cauchy problem (4.14) belongs to $L^{r_{1}}\left((0, T), \dot{B}_{p_{1}, 2}^{\sigma}\left(\mathbb{R}^{3}\right)\right)$ and satisfies $u=P u$, as well as

$$
\|P u\|_{L^{r_{1}}\left((0, T), \dot{B}_{p_{1}, 2}^{\sigma}\left(\mathbb{R}^{3}\right)\right)} \leqslant C(T)\left(\left\|P u_{\text {init }}\right\|_{\dot{H}^{\mu}}+\|P f\|_{L^{s_{1}}\left((0, T), \dot{B}_{q_{1}, 2}^{\rho}\left(\mathbb{R}^{3}\right)\right)}\right) .
$$

In the case $p_{1}=2$ (hence $r_{1}=\infty, \sigma=\mu$ ), the function $u=P u$ is even in $C\left([0, T], \dot{H}^{\mu}\left(\mathbb{R}^{3}\right)\right)$.

The result of Proposition 4.6 is false for $r_{1}=2, p_{1}=\infty, s_{1}=1, q_{1}=2$ and $\mu=\rho=1, \sigma=0$. However, it is true when truncating frequencies. We use, for $\lambda>0$, the low frequency cut-off operator $S^{\lambda}$, which is the Fourier multiplier with symbol $\chi_{\lambda}:=\chi(\cdot / \lambda)$, where the cut-off function $\chi \in \mathcal{C}_{c}^{\infty}\left(\mathbb{R}^{d},[0,1]\right)$ takes value 1 when $|\xi| \leqslant 1 / 2$, and 0 when $|\xi| \geqslant 1$. Then, we have:

Proposition 4.7. Under assumption (2.1), there is a non-decreasing function $C$ : $(0, \infty) \rightarrow(0, \infty)$ such that, for all $\lambda, T>0$ and for any $u \in \mathcal{C}\left([0, T), H^{1}\left(\mathbb{R}^{3}\right)\right)$ solution to (4.14),

$$
\begin{aligned}
& \left\|S^{\lambda} P u\right\|_{L^{2}\left((0, T), L^{\infty}\left(\mathbb{R}^{3}\right)\right)} \\
& \leqslant C(T) \sqrt{\ln (1+\lambda T)}\left(\left\|\partial_{x} P u_{\mathrm{init}}\right\|_{L^{2}\left(\mathbb{R}^{3}\right)}+\left\|\partial_{x} P f\right\|_{L^{1}\left((0, T), L^{2}\left(\mathbb{R}^{3}\right)\right)}\right) .
\end{aligned}
$$

Estimate (4.19) is proved in the case where the operator $P$ is $P:=-\Delta$ in [24] Proposition 6.3. Even in this case Estimate (4.19) without the cut-off $S^{\lambda}$ is false $[26,30]$. Proposition 4.7 extends [24]'s result to (smooth) variable coefficients. 
Let us mention that one can deduce from these results some similar estimates for the $\mathbb{R}^{N}$-valued solutions $u$ of wave equations of the form:

$$
\left(\partial_{t}^{2}+A\right) u=f \text { in } \mathbb{R}^{3},\left.\quad \partial_{t}^{v} u\right|_{t=0}=u_{v} \quad \text { for } v=0,1,
$$

where $A:=-a(x) \Delta+\sum_{j=1}^{n} B_{j}(x) \partial_{j}+C(x)$, when $a-1 \in \mathcal{C}_{K}^{\infty}\left(\mathbb{R}^{3}\right), a(x) \geqslant$ $c_{0}>0$ and the $B_{j}$ and $C$ are in $\mathcal{C}_{K}^{\infty}\left(\mathbb{R}^{3}, \mathcal{M}_{N \times N}(\mathbb{R})\right)$. These equations stand for the propagation of waves in an inhomogeneous isotropic compact medium $K \subset \mathbb{R}^{3}$ surrounded by vacuum.

To prove Proposition 4.6 and Proposition 4.7 we use the Lax method, that is an explicit representation of the solution which allows to take advantage of oscillations via the method of stationary phase, for each dyadic block, to get a pointwise dispersive estimate. The final step relies on the $T T^{\star}$ argument and the summation over the dyadic blocks.

This kind of strategy is now very classical. In particular, the $T T^{\star}$ argument is due to Ginibre and Velo [20], and the use of bilinear interpolation was initiated by Keel and Tao [25]. We refer here to the book [3] by Bahouri, Chemin and Danchin for a larger overview of its use and of its consequences. However we did not find Proposition 4.7 in the literature so that we now detail a little bit its proof.

Proof of Proposition 4.6. Let us first remark that it is sufficient to prove Estimate (4.18) for smooth data (by the usual regularization process) and locally in time. More precisely, it suffices to prove that there is a constant $C>0$ and $T_{1}>0$ such that, for all $\lambda>0$ and for all $u \in \mathcal{C}\left(\left[0, T_{1}\right], H^{1}\left(\mathbb{R}^{3}\right)\right)$ solution to (4.14),

$$
\|P u\|_{L^{r_{1}}\left(\left(0, T_{1}\right), \dot{B}_{p_{1}, 2}^{\sigma}\left(\mathbb{R}^{3}\right)\right)} \leqslant C\left(\left\|P u_{\mathrm{init}}\right\|_{\dot{H}^{\mu}}+\|P f\|_{L^{s_{1}}\left(\left(0, T_{1}\right), \dot{B}_{q_{1}, 2}^{\rho}\left(\mathbb{R}^{3}\right)\right)}\right) .
$$

Indeed, apply several times Estimate (4.20) on time intervals of the form $\left(k T_{1},(k+\right.$ 1) $T_{1}$ ), with $k$ an integer ranging from zero to the integral part $K$ of $T / T_{1}$ (plus the interval $\left.\left(K T_{1}, T\right)\right)$ : on the right hand side, $\left\|P u\left(k T_{1}\right)\right\|_{\dot{H}^{\mu}}$ is estimated by $C(\| P u((k-$ 1) $\left.\left.T_{1}\right)\left\|_{\dot{H}^{\mu}}+\right\| P f \|_{L^{s_{1}}\left(\left((k-1) T_{1}, k T_{1}\right), \dot{B}_{q_{1}, 2}^{\rho}\left(\mathbb{R}^{3}\right)\right)}\right)$. Summing up gives (4.18).

Now, consider the operator $S(t):=e^{-t B} P$. It admits a parametrix, and thus is given by a sum $S(t)=I_{+}(t)+I_{-}(t)$ of operators on the dispersive eigenspaces, which are Fourier Integral Operators: for any smooth function $u(x)$,

$$
\left(I_{ \pm}(t) u\right)(x)=\int_{\mathbb{R}^{3} \times \mathbb{R}^{3}} e^{i(\Psi(t, x, \xi)-2 \pi y \cdot \xi)} a(t, x, \xi) u(y) d \xi d y,
$$

and we drop the subscript \pm in the sequel. The phase $\Psi(t, x, \xi)$ is real, positively homogeneous of degree one in $\xi, \mathcal{C}^{\infty}$ for $\xi \neq 0$, and satisfies the eikonal equation:

$$
\partial_{t} \Psi(t, x, \xi)= \pm\left(\kappa_{1} \kappa_{2}(x)\right)^{-1 / 2}\left|\Psi_{x}^{\prime}(t, x, \xi)\right|,
$$


with

$$
\left.\Psi\right|_{t=0}(x, \xi)=2 \pi x \cdot \xi .
$$

The amplitude $a$ is in Hörmander's class $S^{0}$, and admits an asymptotic expansion whose successive orders satisfy a sequence of linear hyperbolic equations. Such a method was initiated by Lax in his pioneering paper [29]. Because of the caustic phenomenon, the lifespan of smooth solutions to Equation (4.21) is limited. However, since $\Psi$ is homogeneous in $\xi$ and the set $K \times S^{2}$ is compact, there exists $T_{1}>0$ such that the solution to (4.21)-(4.22) remains smooth on $\left[-T_{1}, T_{1}\right]$. Let us mention here that Ludwig [33] succeeded in extending Lax' analysis into a globalin-time result. The arguments have been refined thanks to Hörmander's theory of Fourier Integral Operators [17,22]. But we use the parametrices (and the solution operators $I(t)$ ) only locally in time and we refer for their construction to the work of Chazarain [15], Nirenberg and Treves [37] and [36], Kumano-go [27] and Brenner [9]. In particular we refer to the last one for the following precious informations about the phase ([9, Lemma 2.1]): there exist $c, C>0$ such that

(i) $c|\xi| \leqslant\left|\Psi_{x}^{\prime}\right| \leqslant C|\xi|$ on $\left[-T_{1}, T_{1}\right] \times \mathbb{R}^{3} \times\left(\mathbb{R}^{3} \backslash\{0\}\right)$;

(ii) $c I d \leqslant \pm \Psi_{x \xi}^{\prime \prime} \leqslant C I d, \Psi_{x \xi}^{\prime \prime}$ being real symmetric, on $\left[-T_{1}, T_{1}\right] \times \mathbb{R}^{3} \times\left(\mathbb{R}^{3} \backslash\right.$ $\{0\})$

(iii) $\Psi_{\xi \xi}^{\prime \prime}$ is semi-definite with rank 2 for $|\xi| \neq 0, t \neq 0$; and for $|\xi|=1, x \in K$, there is a constant $c_{0}>0$ such that the moduli of the non-zero eigenvalues of $\Psi_{\xi \xi}^{\prime \prime}$ are bounded from below by $c_{0}|t|$;

(iv) for $x \notin K$, the above results are consequence of the exact formula: $\Psi(t, x, \xi)=$ $2 \pi x \cdot \xi \pm 2 \pi\left(\kappa_{1} \kappa_{2}(x)\right)^{-1 / 2} t|\xi|$.

Note that these results imply that the kernel of $I(t)$ is a Lagrangian distribution.

We use the $T T^{\star}$ method for the frequency localized operators

$$
T_{j}(t):=\Delta_{j} I(t), j \in \mathbb{Z} .
$$

The composed operator $T_{j}(t) T_{j}\left(t^{\prime}\right)^{\star}$ is then

$$
T_{j}(t) T_{j}\left(t^{\prime}\right)^{\star}=\Delta_{j} I\left(t-t^{\prime}\right) \Delta_{j} .
$$

Here is the $T T^{\star}$ result.

Lemma 4.8. There exist $0<c<C$ such that for all $j \in \mathbb{Z}, u \in L^{2}\left(\mathbb{R}^{3}\right), p, r \in$ $[1, \infty]$ and $f \in L^{r^{\prime}}\left((0, T), L^{p^{\prime}}\left(\mathbb{R}^{3}\right)\right)$,

$$
\begin{gathered}
\left\|T_{j}(t) u\right\|_{L^{r}\left((0, T), L^{p}\left(\mathbb{R}^{3}\right)\right)} \leqslant\|u\|_{L^{2}\left(\mathbb{R}^{3}\right)} \sup _{g \in \mathcal{B}_{r, p}^{j}}\left(b_{j}(g, g)\right)^{\frac{1}{2}}, \\
\text { and }\left\|\int_{0}^{T} T_{j}(t) T_{j}\left(t^{\prime}\right)^{\star} f\left(t^{\prime}\right) d t^{\prime}\right\|_{L^{r}\left((0, T), L^{p}\left(\mathbb{R}^{3}\right)\right)}=\sup _{g \in \mathcal{B}_{r, p}^{j}}\left|b_{j}(f, g)\right|,
\end{gathered}
$$


with

$$
b_{j}(f, g):=\int_{(0, T) \times(0, T)}\left\langle T_{j}(t) T_{j}\left(t^{\prime}\right)^{\star} f\left(t^{\prime}\right), g(t)\right\rangle_{L^{2}\left(\mathbb{R}^{3}\right)} d t d t^{\prime},
$$

$\mathcal{B}_{r, p}^{j}$ the space of functions $g(t, x)$ in $\mathcal{B}_{r, p}$ whose Fourier transform is supported in $c 2^{j} \leqslant|\xi| \leqslant C 2^{j}$, and $\mathcal{B}_{r, p}$ the space of smooth functions $g(t, x)$ satisfying $\|g\|_{L^{r^{\prime}}\left((0, T), L^{p^{\prime}}\left(\mathbb{R}^{3}\right)\right)} \leqslant 1$.

Proof. Begin with

$$
\left\|T_{j}(t) u\right\|_{L^{r}\left((0, T), L^{p}\left(\mathbb{R}^{3}\right)\right)}=\sup _{g \in \mathcal{B}_{r, p}}\left|\int_{(0, T) \times \mathbb{R}^{3}} T_{j}(t) u(x) \cdot g(t, x) d t d x\right| .
$$

Then, use the Plancherel identity plus the properties of the support of the Fourier transform of $T_{j}(t) u$ to get

$$
\left\|T_{j}(t) u\right\|_{L^{r}\left((0, T), L^{p}\left(\mathbb{R}^{3}\right)\right)}=\sup _{g \in \mathcal{B}_{r, p}^{j}}\left|\int_{(0, T) \times \mathbb{R}^{3}} T_{j}(t) u \cdot g d t d x\right| .
$$

Using Fubini's principle, transposition and Cauchy-Schwarz inequality, observe that

$$
\left\|T_{j}(t) u\right\|_{L^{2}\left((0, T), L^{\infty}\left(\mathbb{R}^{3}\right)\right)} \leqslant\|u\|_{L^{2}\left(\mathbb{R}^{3}\right)} \sup _{g \in \mathcal{B}_{r, p}^{j}}\left\|\int_{0}^{T} T_{j}(t)^{\star} g d t\right\|_{L^{2}\left(\mathbb{R}^{3}\right)} .
$$

Using again Fubini's principle and transposition, get

$$
\left\|\int_{0}^{T} T_{j}(t)^{\star} g(t) d t\right\|_{L^{2}\left(\mathbb{R}^{3}\right)}^{2}=b_{j}(g, g) .
$$

This leads to (4.23). Moreover,

$$
\begin{aligned}
& \left\|\int_{0}^{T} T_{j}(t) T_{j}\left(t^{\prime}\right)^{\star} f\left(t^{\prime}\right) d t^{\prime}\right\|_{L^{r}\left((0, T), L^{p}\left(\mathbb{R}^{3}\right)\right)} \\
& =\sup _{g \in \mathcal{B}_{r, p}^{j}}\left|\int_{(0, T) \times \mathbb{R}^{3}}\left(\int_{0}^{T} T_{j}(t) T_{j}\left(t^{\prime}\right)^{\star} f\left(t^{\prime}\right) d t^{\prime}\right) \cdot g(t) d t d x\right| \\
& =\sup _{g \in \mathcal{B}_{r, p}^{j}}\left|b_{j}(f, g)\right| .
\end{aligned}
$$

Now, we need to estimate $b_{j}(f, g)$. We start with a pointwise estimate. 
Lemma 4.9. There exists $C>0$ such that for all $j \in \mathbb{Z},\left(t, t^{\prime}\right) \in(0, T) \times(0, T)$ and $u \in L^{1}\left(\mathbb{R}^{3}\right)$,

$$
\left\|T_{j}(t)\left(T_{j}\right)\left(t^{\prime}\right)^{\star} u\right\|_{L^{\infty}\left(\mathbb{R}^{3}\right)} \leqslant C 2^{3 j}\left(1+2^{j}\left|t-t^{\prime}\right|\right)^{-1}\|u\|_{L^{1}\left(\mathbb{R}^{3}\right)} .
$$

Proof. Since $\Delta_{j}$ is a bounded operator on $L^{p}\left(\mathbb{R}^{3}\right)$ for all $p \in[1, \infty]$, it is sufficient to prove that for all $t \in(-T, T)$ and $u \in L^{1}\left(\mathbb{R}^{3}\right)$,

$$
\left\|I(t) \Delta_{j} u\right\|_{L^{\infty}\left(\mathbb{R}^{3}\right)} \leqslant C 2^{3 j}\left(1+2^{j}|t|\right)^{-1}\|u\|_{L^{1}\left(\mathbb{R}^{3}\right)} .
$$

Writing down $I(t) \Delta_{j} u$, we get, for all $x \in \mathbb{R}^{3}$ :

$$
\begin{aligned}
\left(I(t) \Delta_{j} u\right)(x) & =\int_{\mathbb{R}^{3} \times \mathbb{R}^{3}} e^{i(\Psi(t, x, \xi)-2 \pi y \cdot \xi)} a(t, x, \xi) \varphi\left(2^{-j} D\right) u(y) d \xi d y \\
& =\int_{\mathbb{R}^{3}} e^{i \Psi(t, x, \xi)} a(t, x, \xi) \varphi\left(2^{-j} \xi\right) \hat{u}(\xi) d \xi \\
& =2^{3 j} \int_{\mathbb{R}^{3}} e^{i 2^{j} \Psi(t, x, \eta)} a\left(t, x, 2^{j} \eta\right) \varphi(\eta) \hat{u}\left(2^{j} \eta\right) d \eta
\end{aligned}
$$

so that

$$
\left|\left(I(t) \Delta_{j} u\right)(x)\right| \leqslant 2^{3 j} \sup _{y \in \mathbb{R}^{3}}\left|\int_{\mathbb{R}^{3}} e^{i 2^{j}(\Psi(t, x, \eta)-y \cdot \eta)} a\left(t, x, 2^{j} \eta\right) \varphi(\eta) d \eta\right|\|u\|_{L^{1}\left(\mathbb{R}^{3}\right)} .
$$

To get (4.27), simply apply the following lemma of stationary phase.

Lemma 4.10 (Littman [31]). Let $\Psi(\xi)$ be a real function $\mathcal{C}^{\infty}$ such that the rank of its Hessian matrix $\Psi_{\xi \xi}^{\prime \prime}$ is at least $\rho$ and let $v(\xi)$ be a function supported in a ring. Then there exists $M \in \mathbb{N}$ and $C>0$ (which depends only on a finite number of derivatives of $\Psi$, of a lower bound of the maximum of the abolute values of the minors of order $\rho$ of $\Psi_{\xi \xi}^{\prime \prime}$, on supp $v$ ) such that, for all $\Lambda \in \mathbb{R}$,

$$
\left\|\mathcal{F}^{-1}\left(e^{i \Lambda \Psi} v\right)\right\|_{\left.L^{\infty}\left(\mathbb{R}^{3}\right)\right)} \leqslant C(1+|\Lambda|)^{-\frac{\rho}{2}} \cdot \sum_{|\alpha| \leqslant M}\left\|D^{\alpha} v\right\|_{L^{1}\left(\mathbb{R}^{3}\right)} .
$$

To use this lemma, distinguish between short times, for which the eikonal equation (4.21) implies that the phase $\Psi$ admits the expansion

$$
\Psi(t, x, \xi)=2 \pi\left(x \cdot \xi \pm t\left(\kappa_{1} \kappa_{2}(x)\right)^{-1 / 2}|\xi|\right)+O\left(t^{2}\right),
$$

(thus $\rho=2, \Lambda=2^{j}\left(\kappa_{1} \kappa_{2}(x)\right)^{-1 / 2} t$ is suitable), and subsequent times, for which Estimate (4.3) on the phase gives $\rho=2$ (and $\Lambda=2^{j}$ ). This yields

$$
\sup _{y \in \mathbb{R}^{3}}\left|\int_{\mathbb{R}^{3}} e^{i 2^{j}(\Psi(t, x, \eta)-y \cdot \eta)} a\left(t, x, 2^{j} \eta\right) \varphi(\eta) d \eta\right| \leqslant C\left(1+2^{j} t\right)^{-1} .
$$


Since $T_{j}(t) T_{j}\left(t^{\prime}\right)^{\star}$ is also bounded on $L^{2}\left(\mathbb{R}^{3}\right)$, from the above result and the RieszThorin Interpolation Theorem, we infer the following.

Lemma 4.11. There exists $C>0$ such that for all $j \in \mathbb{Z},\left(t, t^{\prime}\right) \in(0, T) \times(0, T)$, $p \in[2, \infty]$ and $u \in L^{p^{\prime}}\left(\mathbb{R}^{3}\right)$,

$$
\left\|T_{j}(t) T_{j}\left(t^{\prime}\right)^{\star} u\right\|_{L^{p}\left(\mathbb{R}^{3}\right)} \leqslant C 2^{3 j(1-2 / p)}\left(1+2^{j}\left|t-t^{\prime}\right|\right)^{-(1-2 / p)}\|u\|_{L^{p^{\prime}}\left(\mathbb{R}^{3}\right)} .
$$

Lemma 4.12. For any $p_{1}, p_{2} \in[2, \infty)$, define $r_{1}$ and $r_{2}$ by $1 / r_{1}+1 / p_{1}=1 / 2$ and $1 / r_{2}+1 / p_{2}=1 / 2$. Then, there exists $C>0$ such that for all $j \in \mathbb{Z}$, $f \in L^{r_{1}^{\prime}}\left((0, T), L^{p_{1}^{\prime}}\left(\mathbb{R}^{3}\right)\right)$ and $g \in L^{r_{2}^{\prime}}\left((0, T), L^{p_{2}^{\prime}}\left(\mathbb{R}^{3}\right)\right)$,

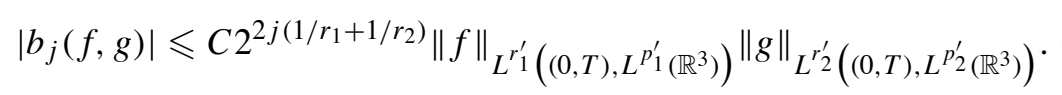

Proof. Using (4.29), we apply Hölder's inequality to get

$$
\left|b_{j}(f, g)\right| \leqslant C 2^{6 j / r_{1}}\left\|\left(\left(1+2^{j}|\cdot|\right)^{-2 / r_{1}} \star\|f\|_{L^{p_{1}^{\prime}\left(\mathbb{R}^{3}\right)}}\right)\left(t^{\prime}\right)\right\| g\left(t^{\prime}\right)\left\|_{L^{p_{1}^{\prime}\left(\mathbb{R}^{3}\right)}}\right\|_{L^{1}(0, T)} .
$$

Using again Hölder's inequality, we get

$$
\begin{aligned}
& \left|b_{j}(f, g)\right| \\
& \leqslant C 2^{6 j / r_{1}}\left\|\left(\left(1+2^{j}|\cdot|\right)^{-2 / r_{1}} \star\|f\|_{L^{p_{1}^{\prime}\left(\mathbb{R}^{3}\right)}}\right)\left(t^{\prime}\right)\right\|_{L^{r_{1}(0, T)}}\|g\|_{L^{r_{1}^{\prime}}\left((0, T), L^{p_{1}^{\prime}\left(\mathbb{R}^{3}\right)}\right)} .
\end{aligned}
$$

Thus, the Hardy-Littlewood-Sobolev inequality implies

$$
\left|b_{j}(f, g)\right| \leqslant C 2^{4 j / r_{1}}\|f\|_{L^{r_{1}^{\prime}}\left((0, T), L^{\left.p_{1}^{\prime}\left(\mathbb{R}^{3}\right)\right)}\right.}\|g\|_{L^{r_{1}^{\prime}}\left((0, T), L^{p_{1}^{\prime}}\left(\mathbb{R}^{3}\right)\right)} .
$$

Moreover, (4.26) yields

$$
\left\|\int_{0}^{T} T_{j}(t)^{\star} f(t) d t\right\|_{L^{2}\left(\mathbb{R}^{3}\right)}=b_{j}(f, f)^{1 / 2} \leqslant C 2^{2 j / r_{1}}\|f\|_{L^{r_{1}^{\prime}}\left((0, T), L^{p_{1}^{\prime}\left(\mathbb{R}^{3}\right)}\right)} .
$$

Now, we observe that

$$
b_{j}(f, g)=\int_{0}^{T}\left\langle\int_{0}^{T} T_{j}\left(t^{\prime}\right)^{\star} f\left(t^{\prime}\right) d t^{\prime}, T_{j}(t)^{\star} g(t)\right\rangle_{L^{2}\left(\mathbb{R}^{3}\right)} d t .
$$

We use the Cauchy-Schwarz and the uniform boundedness of the $T_{j}(t)^{\star}$, for $t$ in $(0, T)$, in $L^{2}\left(\mathbb{R}^{3}\right)$ to get

$$
\left|b_{j}(f, g)\right| \leqslant b_{j}(f, f)^{1 / 2}\|g\|_{L^{1}\left((0, T), L^{2}\left(\mathbb{R}^{3}\right)\right)} .
$$

Using now Equation (4.32) yields

$$
\left|b_{j}(f, g)\right| \leqslant C 2^{2 j / r_{1}}\|f\|_{L^{r_{1}^{\prime}}\left((0, T), L^{p_{1}^{\prime}}\left(\mathbb{R}^{3}\right)\right)}\|g\|_{L^{1}\left((0, T), L^{2}\left(\mathbb{R}^{3}\right)\right)} .
$$

Interpolating between (4.31) and (4.34), we find (4.30). 
To complete the proof of Proposition 4.6, we need to sum over all frequencies. Apply Duhamel's principle to get

$$
P u(t)=e^{-t B} P u_{\text {init }}+\int_{0}^{t} e^{\left(t^{\prime}-t\right) B} P f\left(t^{\prime}\right) d t^{\prime} .
$$

The first term in $\Delta_{j} P u(t)$ is thus

$$
\Delta_{j} I(t) u_{\text {init }}=\sum_{k \in \mathbb{Z}} \Delta_{j} I(t) \Delta_{k} u_{\text {init }} .
$$

An important fact in order to estimate $\Delta_{j} I(t) u_{\text {init }}$ is that the quasi-orthogonality of the dyadic blocks is not destroyed by the parametrix $I(t)$. Actually, according to [9, Proposition 1.1 and Lemma 1.4], there exists $L \in \mathbb{N}^{*}$ such that for all $j \in \mathbb{Z}$,

$$
\Delta_{j} I(t)=\sum_{|j-k| \leqslant L} \Delta_{j} I(t) \Delta_{k}+R_{j}(t),
$$

where $R(t):=\sum_{j \in \mathbb{Z}} R_{j}(t)$ satisfies

$$
\|R(t) v\|_{L^{r_{1}\left((0, T), \dot{B}_{p_{1}, 2}^{\sigma}\left(\mathbb{R}^{3}\right)\right)}} \leqslant C\|v\|_{\dot{H}^{\mu}} .
$$

This shows that there is $C>0$ such that

$$
\left\|\Delta_{j} I(t) u_{\text {init }}\right\|_{L^{p_{1}\left(\mathbb{R}^{3}\right)}} \leqslant C\left\|\Delta_{j} I(t) \Delta_{j} u_{\text {init }}\right\|_{L^{p_{1}\left(\mathbb{R}^{3}\right)}}+\left\|R_{j}(t) u_{\text {init }}\right\|_{L^{p_{1}\left(\mathbb{R}^{3}\right)}},
$$

so that

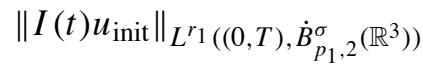

$$
\begin{aligned}
& \leqslant C\|\|\left(2^{j \sigma}\left\|\Delta_{j} I(t) \Delta_{j} u_{\text {init }}\right\|_{\left.L^{p_{1}\left(\mathbb{R}^{3}\right)}\right)}\left\|_{l^{2}(\mathbb{Z})}\right\|_{L^{r_{1}(0, T)}}\right. \\
& +\|\|\left(2^{j \sigma}\left\|R_{j}(t) u_{\text {init }}\right\|_{\left.L^{p_{1}\left(\mathbb{R}^{3}\right)}\right)}\left\|_{l^{2}(\mathbb{Z})}\right\|_{L^{r_{1}(0, T)}}\right.
\end{aligned}
$$

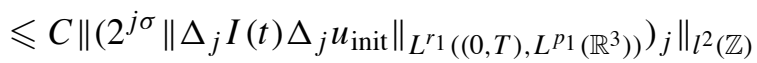

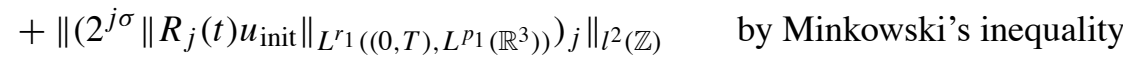

$$
\begin{aligned}
& \leqslant C\left\|\left(2^{j\left(\sigma+2 / r_{1}\right)}\left\|\Delta_{j} u_{\text {init }}\right\|_{L^{2}\left(\mathbb{R}^{3}\right)}\right)_{j}\right\|_{l^{2}(\mathbb{Z})}
\end{aligned}
$$

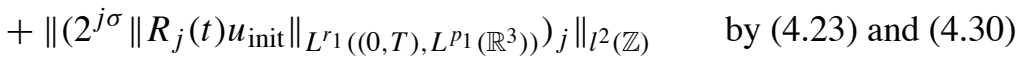

$$
\begin{aligned}
& \leqslant C\left\|u_{\text {init }}\right\|_{\dot{H}^{\mu}} \text { for some new constant } C \text {, using } \mu=\sigma+2 / r_{1} \text { and (4.36). }
\end{aligned}
$$

The estimate for $\int_{0}^{t} e^{\left(t^{\prime}-t\right) B} P f\left(t^{\prime}\right) d t^{\prime}$ follows the same lines, using (4.24) instead of (4.23).

To conclude the proof of Proposition 4.6, consider the case $p_{1}=2$. As (4.18) holds, it is easy to see that $u$ is continuous with respect to time: using a smooth approximation $f_{k} \in L^{s_{1}}\left((0, T), \dot{H}^{\mu}\right)$ of $f$, we get $L u_{k}=f_{k}$, and since $s_{1} \geqslant 1$, the usual energy estimates show that $\left(u_{k}\right)_{k}$ is a Cauchy sequence in $C\left([0, T], \dot{H}^{\mu}\right)$. By (4.18), as $f_{k}$ tends to $f$ in $L^{s_{1}}\left((0, T), \dot{B}_{q_{1}, 2}^{\rho}\right), u_{k}$ tends to $u$ in $C\left([0, T], \dot{H}^{\mu}\right)$. 
Proof of Proposition 4.7. Now, in order to prove Proposition 4.7, it suffices to come back to the proof of Lemma 4.12, taking $p_{1}=\infty\left(\right.$ and $\left.r_{1}=2\right)$. Using the standard Young inequality instead of the Hardy-Littlewood-Sobolev inequality leads to

$$
\left|b_{j}(f, g)\right| \leqslant C 2^{2 j} \ln \left(1+2^{j} T\right)\|f\|_{L^{2}\left((0, T), L^{1}\left(\mathbb{R}^{3}\right)\right)}\|g\|_{L^{2}\left((0, T), L^{1}\left(\mathbb{R}^{3}\right)\right)} .
$$

In particular for any $g$ in $L^{2}\left((0, T), L^{1}\left(\mathbb{R}^{3}\right)\right)$, this yields

$$
\left|b_{j}(g, g)\right|^{1 / 2} \leqslant C 2^{j} \sqrt{\ln \left(1+2^{j} T\right)}\|g\|_{L^{2}\left((0, T), L^{1}\left(\mathbb{R}^{3}\right)\right)} .
$$

Then we apply Lemma 4.8 with $(p, r)=(2, \infty)$ and use $(4.33)$, where we commute $f$ and $g$, to estimate the right hand side of (4.24). We find that for any $u \in L^{2}\left(\mathbb{R}^{3}\right)$, for any $f \in L^{1}\left((0, T), L^{2}\left(\mathbb{R}^{3}\right)\right)$,

$$
\begin{gathered}
\left\|T_{j}(t) u\right\|_{L^{2}\left((0, T), L^{\infty}\left(\mathbb{R}^{3}\right)\right)} \leqslant C 2^{j} \sqrt{\ln \left(1+2^{j} T\right)}\|u\|_{L^{2}\left(\mathbb{R}^{3}\right)}, \\
\left\|\int_{0}^{T} T_{j}(t) T_{j}\left(t^{\prime}\right)^{\star} f\left(t^{\prime}\right) d t^{\prime}\right\|_{L^{2}\left((0, T), L^{\infty}\left(\mathbb{R}^{3}\right)\right)} \leqslant C 2^{j} \sqrt{\ln \left(1+2^{j} T\right)}\|f\|_{L^{1}\left((0, T), L^{2}\left(\mathbb{R}^{3}\right)\right)}
\end{gathered}
$$

Then we proceed as in the proof of Proposition 4.6 to sum over the dyadic blocks whose frequencies are below the cut-off parameter $\lambda$, with an extra factor $\sqrt{\ln (1+\lambda T)}$ (and (4.36) holds for $\left.r_{1}=2, p_{1}=\infty\right)$.

\subsection{Propagation of smoothness: proof of Theorem 2.8, case where $\mu \in(0,1)$}

In the case where $\mu \in(0,1)$, Theorem 2.8 is a consequence of the following proposition:

Proposition 4.13. Suppose that $A$ and $g$ satisfy the estimates (4.6)-(4.10), that $\mu \in(0,1)$ and $u_{\text {init }} \in L^{2}\left(\mathbb{R}^{3}, \mathbb{R}^{6}\right)$ with $P u_{\text {init }} \in H^{\mu}\left(\mathbb{R}^{3}, \mathbb{R}^{6}\right)$. Then the unique solution $\mathbf{u} \in C\left([0, \infty), L^{2}\left(\mathbb{R}^{3}\right)\right)$ of (4.1)-(4.2) given by Lemma 4.5 belongs to $C\left([0, \infty), H^{\mu}\left(\mathbb{R}^{3}\right)\right)$.

In order to prove Proposition 4.13 we introduce, for $T>0$, the space

$$
Y^{\mu}(T):=C\left([0, T], H^{\mu}\left(\mathbb{R}^{3}\right)\right) \cap C^{1}\left([0, T], H^{\mu-1}\left(\mathbb{R}^{3}\right)\right) \cap L^{r}\left((0, T), B_{p, 2}^{0}\left(\mathbb{R}^{3}\right)\right),
$$

where $p:=2 /(1-\mu)$ and $r:=2 / \mu$. These indices belong to $(2, \infty)$. We shall also use the space $Z^{\mu}(T):=Z_{1}^{\mu}(T)+Z_{2}^{\mu}(T)$, where

$$
\begin{aligned}
Z_{1}^{\mu}(T) & :=L^{1}\left((0, T), H^{\mu}\left(\mathbb{R}^{3}\right)\right) \cap C\left([0, T], L^{2}\left(\mathbb{R}^{3}\right)\right), \\
Z_{2}^{\mu}(T) & :=\left\{f \in C\left([0, T], L^{2}\left(\mathbb{R}^{3}\right)\right) \cap L^{r}\left((0, T), B_{p, 2}^{-1}\left(\mathbb{R}^{3}\right)\right) \mid\right. \\
\partial_{t} f & \left.\in L^{1}\left((0, T), H^{\mu-1}\left(\mathbb{R}^{3}\right)\right)+L^{s}\left((0, T), B_{q, 2}^{0}\left(\mathbb{R}^{3}\right)\right)\right\},
\end{aligned}
$$

where $q=2 /(2-\mu)$ and $s=2 /(1+\mu)$. These indices belong to $(1,2)$. 
Lemma 4.14. Suppose that $\mu, T \in(0,1)$, and $f \in Z^{\mu}(T)$. Then there exists $C>0$ such that for any $\mathbf{u}_{\text {init }}$ in $H^{\mu}\left(\mathbb{R}^{3}, \mathbb{R}^{6}\right)$ such that $P \mathbf{u}_{\text {init }}=\mathbf{u}_{\text {init }}$, the unique corresponding solution $\mathbf{u} \in C\left([0, \infty), L^{2}\left(\mathbb{R}^{3}\right)\right)$ to $L \mathbf{u}=f$, with $\left.\mathbf{u}\right|_{t=0}=\mathbf{u}_{\text {init }}$, belongs to $Y^{\mu}(T)$ and

$$
\|\mathbf{u}\|_{Y^{\mu}(T)} \leqslant C\left(\left\|\mathbf{u}_{\text {init }}\right\|_{H^{\mu}\left(\mathbb{R}^{3}\right)}+\|f\|_{Z^{\mu}(T)}\right) .
$$

Proof. By definition $f$ splits into $f=f_{1}+f_{2}$, with $f_{i} \in Z_{i}^{\mu}(T)$, and u into $\mathbf{u}=u_{0}+u_{1}+u_{2}$, where the $u_{i}$ solve the following hyperbolic Cauchy problems:

$$
\begin{array}{lll}
L u_{0}=S_{0} f & \text { with } & \left.u_{0}\right|_{t=0}=S_{0} \mathbf{u}_{\text {init }}, \\
L u_{1}=\left(I d-S_{0}\right) f_{1} & \text { with } & \left.u_{1}\right|_{t=0}=\left(I d-S_{0}\right) \mathbf{u}_{\text {init }}, \\
L u_{2}=\left(I d-S_{0}\right) f_{2} & \text { with } & \left.u_{2}\right|_{t=0}=0 .
\end{array}
$$

We already have by energy estimates that the $u_{i}$ are in $C\left([0, \infty), L^{2}\left(\mathbb{R}^{3}\right)\right)$. Then one gets the estimates of the $u_{i}$ in $C^{1}\left([0, T], H^{\mu-1}\left(\mathbb{R}^{3}\right)\right)$ by using the equations. To get the estimates in $Y^{\mu}(T)$ it therefore only remains to get the estimates in $L^{r}\left((0, T), B_{p, 2}^{0}\left(\mathbb{R}^{3}\right)\right)$.

For $u_{0}$ this just follows the energy estimate thanks to Bernstein lemma.

In order to estimate $u_{1}$ we apply Proposition 4.6 with $p_{1}=p, r_{1}=r, q_{1}=2$, $s_{1}=1, \sigma=0, \rho=\mu$. This gives the estimate of $u_{1}$ in $L^{r}\left((0, T), B_{p, 2}^{0}\left(\mathbb{R}^{3}\right)\right)$ by the right-hand side of (4.39).

In order to estimate $u_{2}$ we observe that $\partial_{t} u_{2}$ satisfies $L \partial_{t} u_{2}=\left(I d-S_{0}\right) \partial_{t} f_{2}$, with $\left.\partial_{t} u_{2}\right|_{t=0}=\left.\left(I d-S_{0}\right) f_{2}\right|_{t=0}$. By assumption there exists $g_{a} \in L^{1}((0, T)$, $\left.H^{\mu-1}\left(\mathbb{R}^{3}\right)\right)$ and $g_{b} \in L^{s}\left((0, T), B_{q, 2}^{0}\left(\mathbb{R}^{3}\right)\right)$ such that $\partial_{t} f_{2}=g_{a}+g_{b}$. We split accordingly $\partial_{t} u_{2}$ into $\partial_{t} u_{2}=u_{a}+u_{b}$, where $u_{a}$ solves $L u_{a}=g_{a}$, with $\left.u_{a}\right|_{t=0}=$ $\left.\left(I d-S_{0}\right) f_{2}\right|_{t=0}$, and $u_{b}$ solves $L u_{b}=g_{b}$, with $\left.u_{b}\right|_{t=0}=0$. In order to estimate $u_{a}$ (respectively $u_{b}$ ) we apply Proposition 4.6 with $p_{1}=p, r_{1}=r, q_{1}=2$, $s_{1}=1, \sigma=-1, \rho=\mu-1$ (respectively with $p_{1}=p, r_{1}=r, q_{1}=q$, $s_{1}=s, \sigma=-1, \rho=0$ and $\mu-1$ instead of $\left.\mu\right)$. This yields the estimate of $\partial_{t} u_{2}$ in $L^{r}\left((0, T), B_{p, 2}^{-1}\left(\mathbb{R}^{3}\right)\right)$ by $\left\|f_{2}\right\|_{Z_{2}^{\mu}(T)}$. As a consequence $B u_{2}=-\partial_{t} u_{2}+$ $\left(I d-S_{0}\right) f_{2} \in L^{r}\left((0, T), B_{p, 2}^{-1}\left(\mathbb{R}^{3}\right)\right)$. Since $P u_{2}=u_{2}$ this entails that $u_{2} \in$ $L^{r}\left((0, T), B_{p, 2}^{0}\left(\mathbb{R}^{3}\right)\right)$.

The proof of Proposition 4.13 follows by induction of the following lemma:

Lemma 4.15. Suppose that $A$ and $g$ satisfy the estimates (4.6)-(4.10), and $\mu \in$ $(0,1)$. Then there exists $T_{1}>0$ and $C>0$ such that for any $u_{\text {init }} \in L^{2}\left(\mathbb{R}^{3}, \mathbb{R}^{6}\right)$ with $P u_{\mathrm{init}} \in H^{\mu}\left(\mathbb{R}^{3}, \mathbb{R}^{6}\right)$, the unique corresponding solution $\mathbf{u} \in C\left([0, \infty), L^{2}\left(\mathbb{R}^{3}\right)\right)$ to (4.1)-(4.2) given by Lemma 4.5 belongs to $Y^{\mu}\left(T_{1}\right)$ and

$$
\|\mathbf{u}\|_{Y^{\mu}\left(T_{1}\right)} \leqslant C\left(\left\|P u_{\text {init }}\right\|_{H^{\mu}\left(\mathbb{R}^{3}\right)}+\|g\|_{Z^{\mu}\left(T_{1}\right)}\right) .
$$

Proof. In order to prove Lemma 4.15 we recall the following estimate: 
Lemma 4.16 (Joly-Métivier-Rauch [24, Lemma 5.3]). There is a constant $C$, which depends only on $\|A\|_{L^{\infty}\left((0, T), L^{\infty}\left(\mathbb{R}^{3}\right)\right)},\left\|\partial_{t} A\right\|_{L^{\infty}\left((0, T), L^{2}\left(\mathbb{R}^{3}\right)\right)}$ and $\mu$, such that for all $T \in(0,1)$ and $u \in Y^{\mu}(T)$, Au belongs to $Z^{\mu}(T)$, and

$$
\|A u\|_{Z^{\mu}(T)} \leqslant C T^{\mu / 2}\|u\|_{Y^{\mu}(T)}+C\|u\|_{L^{\infty}\left((0, T), L^{2}\left(\mathbb{R}^{3}\right)\right)} .
$$

Let us warn the reader that there is a small misprint in the right hand side in [24, Lemma 5.3], which is corrected above. We also have:

Lemma 4.17. If $g$ satisfies (4.9)-(4.10), then for any $T>0, g$ belongs to $Z^{\mu}(T)$.

Proof. Since $1<q<2<p<\infty$ there hold continuous embeddings $L^{q} \subset B_{q, 2}^{0}$ and $L^{p} \subset B_{p, 2}^{0} \subset B_{p, 2}^{-1}$, so that, using (4.9)-(4.10), we get, for any $T>0$, that $g$ is in $Z_{2}^{\mu}(T) \subset Z^{\mu}(T)$.

According to Lemma 4.16 and Lemma 4.17 there is a constant $C$ such that for all $T \in(0,1)$, if $\mathbf{u} \in Y^{\mu}(T)$ then $f:=P(A \mathbf{u})+P g \in Z^{\mu}(T)$ and

$$
\|f\|_{Z^{\mu}(T)} \leqslant C T^{\mu / 2}\|\mathbf{u}\|_{Y^{\mu}(T)}+C\|\mathbf{u}\|_{L^{\infty}\left((0, T), L^{2}\left(\mathbb{R}^{3}\right)\right)} .
$$

Therefore applying Lemma 4.14 and choosing $T_{1}$ small enough we get Lemma 4.15 .

\subsection{Propagation of smoothness: proof of Theorem 2.8 (a), case where $\mu=1$}

We now consider $U_{\text {init }}$ in $L_{\text {div }}$ with $\operatorname{curl} u_{\text {init, } i} \in L^{2}\left(\mathbb{R}^{3}\right)$, for $i=1,2$, and we consider $U$ solution to (2.6)-(2.8) with $U_{\text {init }}$ as initial data given by Theorem 2.6. The idea is to estimate $B(P u)=P(A u)+P g-\partial_{t} P u$ from (4.1).

Lemma 4.18. Define A and $g$ by (4.3)-(4.5). The following holds true:

$$
\begin{aligned}
\partial_{t} A & \in L_{\mathrm{loc}}^{\infty}\left((0, \infty), L^{3}\left(\mathbb{R}^{3}\right)\right), \\
\partial_{t} g & \in L_{\mathrm{loc}}^{\infty}\left((0, \infty), L^{2}\left(\mathbb{R}^{3}\right)\right) .
\end{aligned}
$$

Proof. We apply Theorem 2.8 in the case $\mu=1 / 2$. This yields that $u$ belongs to $C\left([0, \infty), H^{1 / 2}\left(\mathbb{R}^{3}\right)\right)$ and thus also to $L_{\text {loc }}^{\infty}\left((0, \infty), L^{3}\left(\mathbb{R}^{3}\right)\right)$. We then infer that $\partial_{t} v \in L_{\mathrm{loc}}^{\infty}\left((0, \infty), L^{3}\left(\mathbb{R}^{3}\right)\right)$ and then we get the estimates (4.43) and (4.43).

Lemma 4.19. Define $A$ and $g$ by (4.3)-(4.5). If $\mathbf{u} \in L_{\mathrm{loc}}^{\infty}\left((0, \infty), H^{1}\left(\mathbb{R}^{3}\right)\right) \cap$ $W_{\text {loc }}^{1, \infty}\left((0, \infty), L^{2}\left(\mathbb{R}^{3}\right)\right)$ then $f:=P(A \mathbf{u}+g)$ satisfies $\partial_{t} f \in L_{\mathrm{loc}}^{\infty}\left((0, \infty), L^{2}\left(\mathbb{R}^{3}\right)\right)$, and for any $T>0$, there exists $C>0$, which only depends on $T, A$ and $g$, such that

$$
\left\|\partial_{t} f\right\|_{L^{\infty}\left((0, T), L^{2}\left(\mathbb{R}^{3}\right)\right)} \leqslant C\left(\|\mathbf{u}\|_{L^{\infty}\left((0, T), H^{1}\left(\mathbb{R}^{3}\right)\right)}+\left\|\partial_{t} \mathbf{u}\right\|_{L^{\infty}\left((0, T), L^{2}\left(\mathbb{R}^{3}\right)\right)}+1\right) .
$$


Proof. We have $\partial_{t} f=P\left(\partial_{t} A \mathbf{u}+A \partial_{t} \mathbf{u}+\partial_{t} g\right)$, so that using Hölder's inequality, the continuous embedding $L^{6}\left(\mathbb{R}^{3}\right) \subset H^{1}\left(\mathbb{R}^{3}\right)$ and the estimates (4.43)-(4.44), we get (4.45).

Now we observe that $\partial_{t} \mathbf{u}$ solves for $x \in \mathbb{R}^{3}$,

$$
\begin{aligned}
\left(\partial_{t}+B\right) \partial_{t} \mathbf{u} & =\partial_{t}(P(A \mathbf{u})+P g), \\
\left.\partial_{t} \mathbf{u}\right|_{t=0} & =-B u_{\text {init }}+P\left(\left.A\right|_{t=0} \mathbf{u}_{\text {init }}\right)+\left.P g\right|_{t=0} .
\end{aligned}
$$

This provides an estimate of $\partial_{t} \mathbf{u}$ in $L_{\mathrm{loc}}^{\infty}\left((0, \infty), L^{2}\left(\mathbb{R}^{3}\right)\right)$, and using (4.1)-(4.2), of $B \mathbf{u}$ in $L_{\mathrm{loc}}^{\infty}\left((0, \infty), L^{2}\left(\mathbb{R}^{3}\right)\right)$, hence of $\mathbf{u}$ in $L_{\mathrm{loc}}^{\infty}\left((0, \infty), H^{1}\left(\mathbb{R}^{3}\right)\right)$.

\subsection{Uniqueness: proof of Theorem 2.8 (b)}

Let us recall that in this section we assume that there exists $j \in\{1,2\}$ such that $l_{3-j} F=0$ and such that $F$ depends only on $\left(x, v, u_{j}\right)$. Let $U_{\text {init }} \in L_{\text {div }}$ with curl $u_{\text {init, } i} \in L^{2}\left(\mathbb{R}^{3}\right)$, for $i=1,2$. Let $U$ and $U^{\prime}$ be two solutions to (2.6)-(2.8), given by Theorem 2.6, both with $U_{\text {init }}$ as initial data. The difference $\delta U:=U^{\prime}-$ $U=(\delta u, \delta v)$ between $U=(u, v)$ and $U^{\prime}=\left(u^{\prime}, v^{\prime}\right)$ is solution to the following hyperbolic system

$$
\begin{aligned}
M(\delta U): & =\left(\left(\partial_{t}+B\right) \delta u, \partial_{t} \delta v\right)=\left(\left(\kappa^{-1} \cdot l\right) \delta F, \delta F\right), \\
\text { where } \quad \delta F & =F\left(x, v^{\prime}, u_{j}^{\prime}\right)-F\left(x, v, u_{j}\right) .
\end{aligned}
$$

Thanks to (2.3) we have

$$
\begin{aligned}
\delta F= & F_{0}\left(x, v^{\prime}, u_{j}^{\prime}\right)-F_{0}\left(x, v, u_{j}\right)+\left(F_{1}\left(x, v^{\prime}\right)-F_{1}(x, v)\right) \cdot u_{j} \\
& +F_{1}\left(x, v^{\prime}\right) \cdot\left(u_{j}-u_{j}^{\prime}\right) .
\end{aligned}
$$

The first and last terms in (4.49) are easily estimated in $L^{2}\left(\mathbb{R}^{3}\right)$ by $C_{F}\left(\left\|v_{0}\right\|_{L^{\infty}\left(\mathbb{R}^{3}\right)} e^{K t}\right)\|\delta U\|_{L^{2}\left(\mathbb{R}^{3}\right)}$. To deal with the second one we construct a $L^{\infty}$ approximation of the field $u_{j}$ (analogous to the ones of [24, Lemma 6.2], and [21, Lemma 2.7]).

Lemma 4.20. There is a non-decreasing function $C:(0, \infty) \rightarrow(0, \infty)$ and for all $T>0$, there exists $\left(u_{\|, j}^{\lambda}\right)_{\lambda \geqslant e} \subset L^{\infty}\left((0, T) \times \mathbb{R}^{3}\right)$ such that for all $\lambda \geqslant e$,

$$
\begin{array}{ll} 
& \left\|u_{\|, j}^{\lambda}(t)\right\|_{L^{\infty}\left((0, T) \times \mathbb{R}^{3}\right)} \leqslant C(T) \ln \lambda, \\
\text { and } & \left\|\left(\left(I d-P_{j}\right) u_{j}-u_{\|, j}^{\lambda}\right)(t)\right\|_{L^{2}\left(\mathbb{R}^{3}\right)} \leqslant C(T) / \lambda, \\
\text { for all } & t \in[0, T], \\
& \left\|S^{\lambda} P_{j} u_{j}\right\|_{L^{2}\left((0, T), L^{\infty}\left(\mathbb{R}^{3}\right)\right)} \leqslant C(T) \sqrt{\ln \lambda}, \\
\text { and } & \|\left(\left(I d-S^{\lambda}\right) P_{j} u_{j}(t) \|_{L^{2}\left(\mathbb{R}^{3}\right)} \leqslant C(T) / \lambda,\right. \\
\text { for all } & t \in[0, T] .
\end{array}
$$


Let us admit for a while Lemma 4.20 in order to finish the proof of Theorem 2.8. Fix $T>0$ and consider $t \in(0, T)$. In the second term on the right-hand side of (4.49), decompose $u_{j}=u_{\|, j}^{\lambda}+\left(\left(I d-P_{j}\right) u_{j}-u_{\|, j}^{\lambda}\right)+S^{\lambda} P_{j} u_{j}+\left(I d-S^{\lambda}\right) P_{j} u_{j}$. We infer from Lemma 4.20 that

$$
\begin{aligned}
& \|\delta F\|_{L^{2}\left(\mathbb{R}^{3}\right)} \leqslant C_{F}\left(\left\|v_{\text {init }}\right\|_{L^{\infty}\left(\mathbb{R}^{3}\right)} e^{K T}\right) \\
& \cdot\left(\|\delta U\|_{L^{2}\left(\mathbb{R}^{3}\right)}+\left(C(T) \ln \lambda+\left\|S^{\lambda} P_{j} u_{j}\right\|_{L^{\infty}\left(\mathbb{R}^{3}\right)}\right)\|\delta v\|_{L^{2}\left(\mathbb{R}^{3}\right)}+2 \frac{C(T)}{\lambda}\|\delta v\|_{L^{\infty}\left(\mathbb{R}^{3}\right)}\right) .
\end{aligned}
$$

The energy estimate, together with Gronwall's Lemma, gives

$$
\|\delta U(t)\|_{L^{2}\left(\mathbb{R}^{3}\right)} \leqslant 2 C_{F} C(T) \frac{t}{\lambda} \exp \left(C_{F} \int_{0}^{t}\left(1+C(T) \ln \lambda+\left\|S^{\lambda} P_{j} u_{j}\left(t^{\prime}\right)\right\|_{L^{\infty}\left(\mathbb{R}^{3}\right)}\right) d t^{\prime}\right) .
$$

Since $\int_{0}^{t}\left(1+C(T) \ln \lambda+\left\|S^{\lambda} P_{j} u_{j}\left(t^{\prime}\right)\right\|_{L^{\infty}\left(\mathbb{R}^{3}\right)}\right) d t^{\prime} \leqslant \bar{C}(T) \ln \lambda$ with $\bar{C}(T) \underset{T \rightarrow 0}{\longrightarrow} 0$, we choose $T_{0}$ small enough (in order to have $\bar{C}\left(T_{0}\right)<1$ ), and let $\lambda$ go to infinity. This shows that $\delta U(t)$ vanishes on $\left[0, T_{0}\right]$. Repeat this procedure on intervals of size $T_{0}$ to get the desired conclusion.

Proof of Lemma 4.20. We define $u_{\|, j}^{\lambda}$ by setting $u_{\|, j}^{\lambda}(t, x):=\left(I d-P_{j}\right) u_{j}(t, x)$ if $\left|\left(I d-P_{j}\right) u_{j}(t, x)\right| \leqslant C \ln \lambda$, and $u_{\|, j}^{\lambda}(t, x):=0$ otherwise, where the constant $C$ is chosen below (independently of $(t, x) \in[0, T] \times \mathbb{R}^{3}$ ). Therefore, for $p \in[2, \infty$ ),

$$
\begin{aligned}
& \left\|\left(\left(I d-P_{j}\right) u_{j}-u_{\|, j}^{\lambda}\right)(t)\right\|_{L^{2}}^{2} \\
& =\int_{\left|\left(I d-P_{j}\right) u_{j}\right| \geqslant C \ln \lambda}\left|\left(I d-P_{j}\right) u_{j}(t)\right|^{2} d x \leqslant(C \ln \lambda)^{2-p}\left\|\left(I d-P_{j}\right) u_{j}(t)\right\|_{L^{p}}^{p} .
\end{aligned}
$$

Now, according to Lemma 4.3, the projection $I d-P$ acts continously in any $L^{p}$ with a norm less than $C_{0} p$. Furthermore, we have

$$
\left\|v_{\text {init }}\right\|_{L^{p}}^{p} \leqslant\left\|v_{\text {init }}\right\|_{L^{\infty}|\Omega|}^{p},
$$

so that $\left(\left\|v_{\text {init }}\right\|_{L^{p}}\right)_{1 \leqslant p \leqslant \infty}$ is bounded. Thus, using Equation (2.6) and the bound from Theorem 2.6 (iii), we infer from (4.53) that

$$
\begin{aligned}
& \left\|\left(\left(I d-P_{j}\right) u_{j}-u_{\|, j}^{\lambda}\right)(t)\right\|_{L^{2}}^{2} \leqslant \frac{\left(C_{0} p e^{K T}\left\|v_{\text {init }}\right\|_{L^{p}}\right)^{p}}{(C \ln \lambda)^{p-2}} \\
& =(C \ln \lambda)^{2} \lambda^{2 \ln \left(2 \frac{C_{0}}{C} e^{K T} \sup _{1 \leqslant q \leqslant \infty}\left\|v_{\text {init }}\right\|_{L^{q}}\right),}
\end{aligned}
$$

choosing $p=2 \ln \lambda$. With $C$ big enough, we obtain (4.50) $(C(T)=$ $2 C_{0} e^{K T+1} \sup _{1 \leqslant q \leqslant \infty}\left\|v_{\text {init }}\right\|_{L^{q}}$ is suitable). 
We are now concerned with the first inequality in (4.51). Coming back to (4.1)-(4.2), the idea is to use the Strichartz estimate (4.19). However, since we are not able to bound $\partial_{x} f$ in $L_{\mathrm{loc}}^{\infty}\left((0, \infty), L^{2}\left(\mathbb{R}^{3}\right)\right)$, we cannot apply (4.19) directly. To overcome this difficulty we introduce some potential vectors. Since $P u_{\text {init }} \in$ $H^{1}\left(\mathbb{R}^{3}\right)$ (respectively since $f=\left(f_{1}, f_{2}\right) \in W_{\text {loc }}^{1, \infty}\left((0, \infty), L^{2}\left(\mathbb{R}^{3}\right)\right)(c f .(4.42)$ and (4.45)) and $P f=f)$, for $i=1,2$, there exists $\phi_{\text {init }}:=\left(\phi_{\text {init }, 1}, \phi_{\text {init }, 2}\right) \in H^{2}\left(\mathbb{R}^{3}\right)$ (respectively $\left.\psi:=\left(\psi_{1}, \psi_{2}\right) \in W_{\text {loc }}^{1, \infty}\left((0, \infty), H^{1}\left(\mathbb{R}^{3}\right)\right)\right)$ such that

$$
\begin{aligned}
\operatorname{div}\left(\kappa_{3-i} \phi_{\text {init }, i}\right)=0, & \operatorname{curl}\left(\phi_{\text {init }, i}\right)=\kappa_{i} u_{\text {init }, i}, \\
\text { (respectively } \operatorname{div}\left(\kappa_{3-i} \psi_{i}\right)=0, & \left.\operatorname{curl}\left(\psi_{i}\right)=\kappa_{i} f_{i}\right) .
\end{aligned}
$$

We consider the operator $\check{B}$ defined by

$$
\begin{gathered}
\check{B}\left(\phi_{1}, \phi_{2}\right)=\left(\kappa_{2}^{-1} \operatorname{curl} \phi_{2},-\kappa_{1}^{-1} \operatorname{curl} \phi_{1}\right) \\
\text { for } \phi:=\left(\phi_{1}, \phi_{2}\right) \in D(\check{B}):=D(B)=H_{\text {curl }} \times H_{\text {curl }} .
\end{gathered}
$$

The operator $\check{B}$ is simply deduced from $B$ by switching $\kappa_{1}$ and $\kappa_{2}$. It therefore shares the same properties and estimates. In addition it satisfies the identity:

$$
\left[\begin{array}{ccc}
\kappa_{1}^{-1} & \text { curl } & 0 \\
& 0 & \kappa_{2}^{-1} \text { curl }
\end{array}\right] \check{B}=B\left[\begin{array}{ccc}
\kappa_{1}^{-1} & \text { curl } & 0 \\
& 0 & \kappa_{2}^{-1} \text { curl }
\end{array}\right]
$$

Let $\phi:=\left(\phi_{1}, \phi_{2}\right)$ be the solution (for $\left.x \in \mathbb{R}^{3}\right)$ of

$$
\left(\partial_{t}+\check{B}\right) \phi=\psi, \quad \text { with }\left.\phi\right|_{t=0}=\phi_{\text {init }} .
$$

Using the identity (4.56) and Lemma 4.5 we obtain

$$
\operatorname{curl}\left(\phi_{i}\right)=\kappa_{i} u_{i}, \quad \text { for } i=1,2 \text {. }
$$

Now observe that $\partial_{t} \phi$ verifies $\left(\partial_{t}+\check{B}\right) \partial_{t} \phi=\partial_{t} \psi$, with $\left.\partial_{t} \phi\right|_{t=0}=\left.\psi\right|_{t=0}-\check{B} \phi_{\text {init }}$. Applying the Strichartz estimate (4.19) we obtain

$$
\begin{aligned}
& \left\|S^{\lambda} \partial_{t} \phi\right\|_{L^{2}\left((0, T), L^{\infty}\left(\mathbb{R}^{3}\right)\right)} \\
& \leqslant C(T) \sqrt{\ln (1+\lambda T)}\left(\left\|\left.\partial_{x} \partial_{t} \phi\right|_{t=0}\right\|_{L^{2}\left(\mathbb{R}^{3}\right)}+\left\|\partial_{t} \partial_{x} \psi\right\|_{L^{1}\left((0, T), L^{2}\left(\mathbb{R}^{3}\right)\right)}\right) .
\end{aligned}
$$

From the definitions of $\phi$ and $\psi$ and from the estimates (4.42) and (4.45) of the previous sections we get :

$$
\left\|\left.\partial_{x} \partial_{t} \phi\right|_{t=0}\right\|_{L^{2}\left(\mathbb{R}^{3}\right)}+\left\|\partial_{t} \partial_{x} \psi\right\|_{L^{1}\left((0, T), L^{2}\left(\mathbb{R}^{3}\right)\right)} \leqslant C(T)\left\|P u_{\text {init }}\right\|_{H^{1}\left(\mathbb{R}^{3}\right)} .
$$

Using now (4.57), observing that $f_{3-j}$ and therefore $\psi_{3-j}$ vanish because of Assumption 2.12, and using (4.58), we obtain the first inequality in (4.51). The second one follows by applying Bernstein lemma. 


\section{Generic uniqueness: proof of Theorem $\mathbf{2 . 9}$}

We apply the following general result of generic uniqueness for evolution equations by Saint-Raymond.

Theorem 5.1 (Saint-Raymond [40, Theorem 1]). Let $\mathcal{E}_{\text {init }}$ be a topological space and $\mathcal{E}$ a metric space. Let $(S)$ be an evolution equation admitting a solution in $\mathcal{E}$ for any initial data in $\mathcal{E}_{\text {init }}$. Consider the following hypotheses.

(H1) For any initial data $U_{\text {init }} \in \mathcal{E}_{\text {init }}$, for any $\left(U_{\text {init }}^{\varepsilon}\right)_{\varepsilon}$ tending to $U_{\text {init }}$ in $\mathcal{E}_{\text {init }}$, for any $\left(U^{\varepsilon}\right)_{\varepsilon}$ in $\mathcal{E}$ respective solutions to $(S)$ with $U_{\text {init }}^{\varepsilon}$ as initial data,

(i) there exists a limit point of $\left(U^{\varepsilon}\right)_{\mathcal{E}}$ in $\mathcal{E}$;

(ii) any limit point of $\left(U^{\varepsilon}\right)_{\mathcal{E}}$ in $\mathcal{E}$ is solution to $(S)$ with $U_{\text {init }}$ as initial data.

(H2) There exists $\mathcal{D}$, dense subset of $\mathcal{E}$, such that for any $U_{\text {init }}$ in $\mathcal{D}$, there exists only one solution to $(S)$ in $\mathcal{E}$ with $U_{\text {init }}$ as initial data.

Under these two hypotheses, there exists a $G_{\delta}$ dense $\widetilde{\mathcal{E}_{\text {init }}}$ of $\mathcal{E}_{\text {init }}$ such that for any $U_{\text {init }} \in \widetilde{\mathcal{E}_{\text {init }}}$, there exists only one solution to $(S)$ in $\mathcal{E}$ with $U_{\text {init }}$ as initial data.

Recall that we denote by $\tau_{s}$ and $\tau_{w}$ respectively the strong and weak topologies of $L^{2}\left(\mathbb{R}^{3}, \mathbb{R}^{6}\right)$, and by $\tilde{\tau}_{s}$ the strong topology of $L^{2}\left(\Omega, \mathbb{R}^{d}\right)$. We consider the product topology $\tau_{s s}$ (respectively $\tau_{w s}$ ) on $\mathbf{L}^{2}$ obtained from $\tau_{s}$ (respectively $\tau_{w}$ ) and $\tilde{\tau}_{s}$.

For any $C_{\text {init }}>0$, consider

$$
\mathcal{E}_{\text {init }}:=\left\{U_{\text {init }} \in L_{\text {div }} \mid\left\|v_{\text {init }}\right\|_{L^{\infty}(\Omega)} \leqslant C_{\text {init }}\right\},
$$

endowed with the topology $\tau_{s s}$ (respectively $\tau_{w s}$ ) inherited from $\mathbf{L}^{2}$, and

$$
\begin{aligned}
& \mathcal{E}:=\left\{U \in C\left([0, \infty), \mathbf{L}^{2}\right)\right. \text {, satisfying (2.9) } \\
& \text { and the estimates (i), (ii), (iii) of Theorem 2.6\}, }
\end{aligned}
$$

endowed with the strong topology (respectively the weak $*$ topology relative to $\tau_{w s}$ ) of $C\left([0, \infty), \mathbf{L}^{2}\right)$. Hypothesis (H1) is a direct consequence of the stability property stated in Theorem 2.6 (respectively Proposition 2.7 ). Now, set

$$
\mathcal{D}:=\left\{U_{\text {init }} \in L_{\text {div }} \text { with } \operatorname{curl} u_{\text {init }, i} \in L^{2}\left(\mathbb{R}^{3}\right) \text {, for } i=1,2\right\},
$$

which is dense in $\mathcal{E}_{\text {init }}$ for the topology $\tau_{s s}$ inherited from $\mathbf{L}^{2}$. Moreover Theorem 2.8 yields that Hypothesis (H2) is satisfied. We can therefore apply Theorem 5.1, what proves Theorem 2.9. 


\section{Quasi-stationary limits: proof of Theorem 2.10, Proposition 2.11 and Theorem 2.12}

Proof of Theorem 2.10. We first observe that the bounds (i), (ii) given by Theorem 2.6 for $U^{\eta}$ are uniform in $\eta \in(0,1)$. Therefore, up to a subsequence, $U^{\eta}$ converges to $U:=(u, v)$ in $W_{\text {loc }}^{1, \infty}\left((0, \infty), L^{2}(\Omega)\right)$ weak $*$ and $v^{\eta}$ converges to $v$ in $L_{\mathrm{loc}}^{\infty}\left((0, \infty), L^{\infty}(\Omega)\right)$ weak $*$. In addition, there holds $B u^{\eta}=\partial_{t} D^{\eta}$, with $D^{\eta}:=-\eta\left(u^{\eta}-\left(\kappa^{-1} \cdot l\right) \overline{v^{\eta}}\right)$. Passing to the limit already yields that $U$ satisfies the (linear) equations (2.6) and $B u=0$. Using Proposition 2.7, we also get that $U$ satisfies (2.8), which means that $v$ solves (2.15).

Proof of Proposition 2.11. The proof is very similar to the uniqueness proof in Theorem 2.8 (b): it relies on some $L^{\infty}$ approximation of $(I d-P)\left(\kappa^{-1} \cdot l\right) v$. Consider $v_{1}, v_{2} \in C\left([0, \infty), L^{2}(\Omega)\right) \cap L_{\mathrm{loc}}^{\infty}\left((0, \infty), L^{\infty}(\Omega)\right)$, solutions to (2.15) with the same initial data $v_{\text {init }}$, and define $\delta v:=v_{1}-v_{2}$. Fix $T>0$. From the properties (2.3)-(2.5) of $F$, we get the pointwise estimate

$$
\begin{aligned}
& \partial_{t}\left(|\delta v|^{2}\right) \leqslant C_{F}\left(\left(\left|(I d-P)\left(\kappa^{-1} \cdot l\right) v_{1}\right|+1\right)|\delta v|^{2}+\left|(I d-P)\left(\kappa^{-1} \cdot l\right) \delta v\right||\delta v|\right) \\
& \text { on }[0, T] \times \Omega,
\end{aligned}
$$

for some constant $C_{F}=C_{F}\left(\left\|v_{\text {init }} e^{K T}\right\|_{L^{\infty}(\Omega)}\right)$. Now, defining for $M>0$

$$
w_{\|}^{M}:=\mathbf{1}_{\left|(I d-P)\left(\kappa^{-1} \cdot l\right) v_{1}\right| \leqslant M}(I d-P)\left(\kappa^{-1} \cdot l\right) v_{1},
$$

we get from Lemma 4.20 that there is $C(T)>0$ such that

$$
\begin{aligned}
& \forall M \geqslant 1, \quad\left\|w_{\|}^{M}\right\|_{L^{\infty}((0, T) \times \Omega)} \leqslant M, \\
& \left\|(I d-P)\left(\kappa^{-1} \cdot l\right) v_{1}-w_{\|}^{M}\right\|_{L^{\infty}\left((0, T), L^{2}(\Omega)\right)} \leqslant C(T) e^{-M / C(T)} .
\end{aligned}
$$

Integrating (6.1) over $\Omega$, using the Cauchy-Schwarz inequality and increasing the constant $C$ (which is still independent of $M$ ), we obtain

$$
\partial_{t}\left(\|\delta v\|_{L^{2}(\Omega)}^{2}\right) \leqslant C_{F}\left((M+1)\|\delta v\|_{L^{2}(\Omega)}^{2}+C(T) e^{-M / C(T)}\right) .
$$

Then, Gronwall's lemma yields

$$
\forall t \in[0, T], \quad\|\delta v(t)\|_{L^{2}(\Omega)}^{2} \leqslant \frac{C(T)}{M+1} e^{C_{F}(M+1) T-M / C(T)} .
$$

Now, choose $T$ so small that $C_{F} M T-M / C(T)<0$ (which is possible, since $C$ is a non-decreasing function of $T$ ), and let $M$ go to infinity. This shows that $\delta v$ vanishes on $[0, T]$. Repeating the argument on successive time intervals yields $v_{1}=v_{2}$. 
Proof of Theorem 2.12. For each $\eta \in(0,1)$, consider a solution $U^{\eta}$ (given by Theorem 2.6) to (2.6), (2.8) and (2.13). Convergence of $v^{\eta}$ (and $\left.(I d-P) u^{\eta}\right)$ is obtained as in the proof of Theorem 2.10 above. Now, drop the index $\eta$ for simplicity. Then, symmetrizing the system by the change of dependent variables

$$
\tilde{u_{i}}=\kappa_{i}^{1 / 2} u_{i}
$$

we get in the distributional sense:

$$
\text { for } i=1,2, \quad \eta \partial_{t} \tilde{u}_{i}+(-1)^{3-i} R_{3-i} \tilde{u}_{3-i}=\eta \kappa_{i}^{-1 / 2} \cdot l_{i} \partial_{t} \bar{v}
$$

and therefore, applying $\partial_{t}$ and combining,

$$
\text { for } i=1,2, \quad \eta^{2} \partial_{t}^{2} \tilde{u_{i}}+R_{i}^{\star} R_{i} \tilde{u_{i}}=(-1)^{i} \eta R_{3-i} \kappa_{3-i}^{-1 / 2} l_{3-i} \partial_{t} \bar{v}+\eta^{2} \kappa_{i}^{-1 / 2} l_{i} \partial_{t}^{2} \bar{v}
$$

where we have set

$$
i=1,2, \quad R_{i}:=\kappa_{3-i}^{-1 / 2} \operatorname{curl} \kappa_{i}^{-1 / 2}\left(=R_{3-i}^{\star}, \text { for the duality in } L^{2}\left(\mathbb{R}^{3}, d x\right)\right) .
$$

System (6.2) shall be understood as a system of wave equations for the "divergence free" parts $\pi_{i} \tilde{u_{i}}$, when

$$
\text { for } i=1,2, \quad \pi_{i}:=\kappa_{i}^{1 / 2} P_{i} \kappa_{i}^{-1 / 2} \text {. }
$$

Then, $\pi=\left(\pi_{1}, \pi_{2}\right)$ is an orthogonal projector in the space $L^{2}\left(\mathbb{R}^{3}, d x\right) \times L^{2}\left(\mathbb{R}^{3}, d x\right)$. Furthermore, from the description of ran $P_{i}$ and $\operatorname{ran}\left(1-P_{i}\right)$ in (2.2), we deduce that

$$
\text { for } i=1,2, \quad R_{i} \pi_{i}=R_{i} \quad\left(\text { and } \quad \pi_{i} R_{i}^{\star}=R_{i}^{\star} \quad\right. \text { by transposition). }
$$

Thus, we have finally:

$$
\text { for } i=1,2, \quad \eta^{2} \partial_{t}^{2} \pi_{i} \tilde{u_{i}}-Q_{i} \pi_{i} \tilde{u_{i}}=(-1)^{i} \eta R_{3-i} \kappa_{3-i}^{-1 / 2} l_{3-i} \partial_{t} \bar{v}+\eta^{2} \pi_{i} \kappa_{i}^{-1 / 2} l_{i} \partial_{t}^{2} \bar{v}
$$

with

$$
\text { for } i=1,2, \quad Q_{i}:=-R_{i}^{\star} R_{i}+\kappa_{i}^{1 / 2} \nabla\left(\kappa_{i}^{-2} \kappa_{3-i}^{-1} \operatorname{div}\left(\kappa_{i}^{1 / 2} \cdot\right)\right) .
$$

From [43, Lemma 3.10], we know that for $i=1,2$, the differential second-order operator $\left(-Q_{i}\right)$ is a self-adjoint, positive, and elliptic. Thus, with $v \in L_{\text {loc }}^{\infty}((0, \infty)$, $\left.L^{\infty}(\Omega)\right) \cap W_{\text {loc }}^{1, \infty}\left((0, \infty), L^{2}(\Omega)\right)$ given, and for given initial data,

$$
\begin{aligned}
\left.\pi_{i} \tilde{u}_{i}\right|_{t=0} & =\pi_{i} \kappa_{i}^{1 / 2} u_{\text {init }, i} \text { and } \eta\left(\partial_{t} \pi_{i} \tilde{u}_{i}\right)_{\left.\right|_{t=0}} \\
& =\eta \pi_{i} \kappa_{i}^{-1 / 2} l_{i} F\left(\overline{v_{\text {init }}}, u_{\text {init }}\right)+(-1)^{i} R_{3-i} \kappa_{3-i}^{1 / 2} u_{\text {init }, 3-i},
\end{aligned}
$$


the solution $\left(\pi_{1} \tilde{u_{1}}, \pi_{2} \tilde{u_{2}}\right)$ to the linear wave equation system (6.3) is uniquely determined. We recover it via vector potentials: defining

for $i=1,2, \eta^{2} \partial_{t}^{2} \phi_{i}-Q_{i} \phi_{i}=\eta \pi_{i} \kappa_{i}^{-1 / 2} l_{i} \partial_{t} \bar{v}, \phi_{\left.i\right|_{t=0}}=0, \eta \partial_{t} \phi_{\left.i\right|_{t=0}}=\pi_{i} \tilde{u}_{\left.i\right|_{t=0}}$,

we have

$$
\text { for } i=1,2, \quad \pi_{i} \tilde{u_{i}}=\eta \partial_{t} \phi_{i}+(-1)^{i} R_{3-i} \phi_{3-i} .
$$

The problem (6.4) also determines uniquely the vector potentials $\phi_{i}$. Since $\pi_{i} \phi_{i}$ also satisfies the problem (6.4) we infer that $\pi_{i} \phi_{i}=\phi_{i}$. Furthermore, [43, Lemma 3.10] ensures that for $i=1,2, Q_{i}$ does not admit 0 as a resonance. One then needs to assume the following:

$$
\text { for } i=1,2, Q_{i} \text { is non-trapping. }
$$

This is enough to apply

Theorem 6.1 (Starynkevitch [43, Theorem 3.2]). Let $Q$ be a non-trapping, $\left(L^{2}\right.$-)self-adjoint, negative, and elliptic differential second-order operator, for which 0 is not a resonance. Let $s>1 / 2, \gamma \in(-3 / 2,1 / 2)$ and $R>0$. Then, there exists $C \geqslant 0$ such that: for all $\left(u_{0}, u_{1}\right) \in \dot{H}_{Q}^{\gamma+1}\left(\mathbb{R}^{3}\right) \times \dot{H}_{Q}^{\gamma}\left(\mathbb{R}^{3}\right)$, and $f$ such that $\langle x\rangle^{s}(-Q)^{\gamma / 2} f \in L^{2}\left((0, \infty) \times \mathbb{R}^{3}\right)$, the solution $u$ to

$$
\partial_{t}^{2} u-Q u=f \text { on }(0, \infty) \times \mathbb{R}^{3}, \quad \text { with } u_{\left.\right|_{t=0}}=u_{0}, \quad \partial_{t} u_{\left.\right|_{t=0}}=u_{1},
$$

satisfies

$$
\begin{aligned}
& \left\|\left(u, \partial_{t} u\right)\right\|_{L^{2}\left((0, \infty), \dot{H}_{Q}^{\gamma+1}\left(B_{R}\right) \times \dot{H}_{Q}^{\gamma}\left(B_{R}\right)\right)} \\
& \leqslant C\left(\left\|u_{0}\right\|_{\dot{H}_{Q}^{\gamma+1}\left(\mathbb{R}^{3}\right)}+\left\|u_{1}\right\|_{\dot{H}_{Q}^{\gamma}\left(\mathbb{R}^{3}\right)}+\left\|\langle x\rangle^{s}(-Q)^{\gamma / 2} f\right\|_{L^{2}\left((0, \infty) \times \mathbb{R}^{3}\right)}\right) .
\end{aligned}
$$

For all $\mu \in \mathbb{R}$, the space $\dot{H}_{Q}^{\mu}\left(\mathbb{R}^{3}\right)$ is defined by the norm

$$
\|v\|_{\dot{H}_{Q}^{\mu}\left(\mathbb{R}^{3}\right)}=\left\|(-Q)^{\mu / 2} v\right\|_{L^{2}\left(\mathbb{R}^{3}\right)} .
$$

We apply the result above to $\phi_{i}(\eta t, x)$, whith $\gamma=0$ and $s=1$. This leads to:

$$
\begin{aligned}
& \forall R>0, \exists C_{R}>0, \\
& \left\|\left(\phi_{i}, \eta \partial_{t} \phi_{i}\right)\right\|_{L^{2}\left((0, \infty), \dot{H}^{1}\left(B_{R}\right) \times L^{2}\left(B_{R}\right)\right)} \leqslant C_{R}\left(\eta^{1 / 2}\left\|\pi_{i} \kappa_{i}^{1 / 2} u_{\text {init }, i}\right\|_{L^{2}\left(\mathbb{R}^{3}\right)}\right. \\
& \left.\quad+\eta\left\|\langle x\rangle \pi_{i} \kappa_{i}^{-1 / 2} l_{i} \partial_{t} \bar{v}\right\|_{L^{2}\left((0, \infty), L^{2}\left(\mathbb{R}^{3}\right)\right)}\right) .
\end{aligned}
$$


The right-hand side is controlled thanks to

Lemma 6.2 (Starynkevitch [43, Lemma 3.11]). For all $R>0$, there exists $C_{R}>$ 0 such that, if $m \in L^{2}\left(\mathbb{R}^{3}\right)$ and $\operatorname{supp}(m) \subset B_{R}$, then

$$
\text { for } i=1,2, \quad\left|\pi_{i} m(x)\right| \leqslant C_{R}\langle x\rangle^{-3}\|m\|_{L^{2}\left(\mathbb{R}^{3}\right)} \quad \text { for a.e. } x \in \mathbb{R}^{3} \text {. }
$$

Since $\pi_{i} \phi_{i}=\phi_{i}$, by the usual $T T^{\star}$ argument, $\left\|R_{i} \phi_{i}\right\|_{L^{2}\left(B_{R}\right)} \leqslant\left\|\phi_{i}\right\|_{\dot{H}^{1}\left(B_{R}\right)}$, and we deduce from (6.5):

$$
\text { for } i=1,2, \quad \pi_{i} \tilde{u_{i}}=O(\sqrt{\eta}) \quad \text { in } L^{2}\left((0, \infty), L_{\mathrm{loc}}^{2}\left(\mathbb{R}^{3}\right)\right),
$$

which yields the convergence of $P u^{\eta}$ to zero in $L^{2}\left((0, \infty), L_{\text {loc }}^{2}\left(\mathbb{R}^{3}\right)\right)$.

\section{References}

[1] F. Alouges and K. BeAuChard, Magnetization switching on small ferromagnetic ellipsoidal samples, ESAIM Control Optim. Calc. Var.15 (2009), 676-711.

[2] F. Alouges and A. SOYEUR, On global weak solutions for Landau Lifschitz equations: existence and non uniqueness, Nonlinear Anal. (11) 18 (1992), 1071-1084.

[3] H. Bahouri, J.-Y. Chemin and R. DAnchin, "Fourier Analysis and Nonlinear Partial Differential Equations", Springer.

[4] J. BERGH and J. LÖFSTRÖM, "Interpolation Spaces. An introduction", Grundlehren der Mathematischen Wissenschaften, No. 223, Springer-Verlag, Berlin, 1976.

[5] B. BidéGArAY-Fesquet, A. BourgeAde and D. ReIGnier, Introducing physical relaxation terms in Bloch equations, J. Comput. Phys. 170 (2001), 603-613.

[6] B. BidÉgARAY-FesqueT, "De Maxwell-Bloch à Schrödinger non Linéaire: une Hiérarchie de Modèles en Optique Quantique", Collection Mathématiques et Applications, Vol. 49, Springer, 2006.

[7] A. BоHM, "Quantum Mechanics", Texts and monographs in Physics, Springer-Verlag, 1979.

[8] R. W. Boyd, "Nonlinear Optics", Academic Press, 1992.

[9] P. BRENNER, On the existence of global smooth solutions of certain semilinear hyperbolic equations, Math. Z. (2) 67 (1979), 99-135.

[10] W. F. BROWN, "Micromagnetics", Interscience Publisher, John Willey and Sons, New York, 1963.

[11] A. P. CALDERÓN and A. Zygmund, On the existence of certain singular integrals, Acta Math. 88 (1952), 85-139.

[12] G. CARBOU and P. FABRIE, Time average in micromagnetism, J. Differential Equations 147 (1998), 383-409.

[13] G. CARBOU and P. FABRIE, Regular solutions for Landau-Lifschitz equation in $\mathbb{R}^{3}$, Commun. Appl. Anal. (1) 5 (2001), 17-30.

[14] G. CARBOU and P. FABRIE, Regular Solutions for Landau-Lifschitz Equation in a Bounded Domain, Differential Integral Equations 14 (2001), 219-229.

[15] J. ChaZARAIn, Opérateurs hyperboliques a caractéristiques de multiplicité constante, Ann. Inst. Fourier (Grenoble) (1) 24 (1974), 173-202.

[16] C. Cohen-Tannoudi, J. Dupont-Roc and G. Grynberg, "Processus d'Interaction Entre Photons et Atomes", Savoirs actuels, Intereditions/Editions du CNRS, 1988.

[17] J. J. DuistermaAT and L. Hörmander, Fourier integral operators, II, Acta Math. (3-4) 128 (1972), 183-269. 
[18] E. Dumas, Global existence for Maxwell-Bloch systems, J. Differential Equations (2) 219 (2005), 484-509.

[19] L. GÅRDING, Hyperbolic equations in the twentieth century, In: "Matériaux pour l'Histoire des Mathématiques au XX ${ }^{\mathrm{e}}$ siècle" (Nice, 1996), Vol. 3 of Sémin. Congr., pages 37-68, Soc. Math. France, Paris, 1998.

[20] J. Ginibre and G. Velo, Generalized Strichartz inequalities for the wave equation, J. Funct. Anal. (1) 133 (1995), 50-68.

[21] H. Haddar, "Modèles Asymptotiques en Ferromagnétisme: Couches Minces et Homogénéisation", Thèse INRIA-École Nationale des Ponts et Chaussées, 2000.

[22] L. Hörmander, Fourier integral operators, I, Acta Math. (1-2) 127 (1971), 79-183.

[23] F. JOCHMANN, Existence of solutions and a quasi-stationary limit for a hyperbolic system describing ferromagnetism, SIAM J. Math. Anal. (2) 34 (electronic) (2002), 315-340.

[24] J. L. Joly, G. MÉTIVIER and J. RaUCH, Global solutions to Maxwell equations in a ferromagnetic medium, Ann. Henri Poincaré (2) 1 (2000), 307-340.

[25] M. KeEL and T. TAO, Endpoint Strichartz estimates, Amer. J. Math. (5) 120 (1998), 955980.

[26] S. Klainerman and M. Machedon, Space-time estimates for null forms and the local existence theorem, Comm. Pure Appl. Math. (9) 46 (1993), 1221-1268.

[27] H. Kumano-GO, A calculus of Fourier integral operators on $R^{n}$ and the fundamental solution for an operator of hyperbolic type, Comm. Partial Differential Equations (1) 1 (1976), 1-44.

[28] L. Landau and E. Lifschitz, "Électrodynamique des milieux continus", Cours de physique théorique, t. 8, Mir, Moscou, 1969.

[29] P. D. LAX, Asymptotic solutions of oscillatory initial value problems, Duke Math. J. 24 (1957), 627-646.

[30] H. LindBlad, Counterexamples to local existence for semi-linear wave equations, Amer. J. Math. (1) 118 (1996), 1-16.

[31] W. LitTMAn, Fourier transforms of surface-carried measures and differentiability of surface averages, Bull. Amer. Math. Soc. 69 (1963), 766-770.

[32] R. Loudon, "The Quantum Theory of Light", Clarendon Press, Oxford, 1991.

[33] D. Ludwig, Exact and asymptotic solutions of the Cauchy problem, Comm. Pure Appl. Math. 13 (1960), 473-508.

[34] G. MÉTIVIER, The Cauchy problem for semilinear hyperbolic systems with discontinuous data, Duke Math. J. (4) 53 (1986), 983-1011.

[35] A. C. Newell and J. V. Moloney, "Nonlinear Optics", Addison-Wesley Publishing Company Advanced Book Program, Redwood City, CA, 1992.

[36] L. NIRENBERG and F. TRÈVES, On local solvability of linear partial differential equations, II, Sufficient conditions, Comm. Pure Appl. Math. 23 (1970), 459-509.

[37] L. Nirenberg and F. Treves, A correction to: On local solvability of linear partial differential equations, II, Sufficient conditions, (Comm. Pure Appl. Math. 23 (1970), 459509). Comm. Pure Appl. Math. (2) 24 (1971), 279-288.

[38] R. Pantell and H. Puthoff, "Fundamentals of Quantum Electronics", Wiley and Sons Inc., N.Y., 1969.

[39] J. RAUCH and M. C. REED, Discontinuous progressing waves for semilinear systems, Comm. Partial Differential Equations (9) 10 (1985), 1033-1075.

[40] L. SAINT-RAYMOND, Un résultat générique d'unicité pour les équations d'évolution, Bull. Soc. Math. France (1) 130 (2002), 87-99.

[41] M. Sargent, M. O. Scully and W. E. Lamb, "Laser Physics", Addison-Wesley, 1977.

[42] J. STARYNKÉVITCH, Local energy estimates for Maxwell-Landau-Lifshitz system and applications, J. Hyperbolic Differ. Equ. (3) 2 (2005), 565-594. 
[43] J. Starynkévitch, "Problèmes d'asymptotique en temps en ferromagnétisme", PhD Thesis.

[44] F. SuEUR, Approche visqueuse de solutions discontinues de systèmes hyperboliques semilinéaires, Ann. Inst. Fourier (Grenoble) (1) 56 (2006), 183-245.

[45] A. Visintin, On Landau-Lifshitz equations for ferromagnetism, Japan J. Appl. Math. (1) 2 (1985), 69-84.

[46] V. I. Judovič, Some bounds for solutions of elliptic equations, Mat. Sb. (N.S.) (101) (suppl.) 59 (1962), 229-244.

Université Grenoble 1

Institut Fourier

100 , rue des mathématiques

BP 74 - 38402 Saint Martin d'Hères, France

edumas@ujf-grenoble.fr

Laboratoire Jacques-Louis Lions

Université Pierre et Marie Curie

Paris 6

75252 Paris, France

fsueur@ann.jussieu.fr 